Nouvelles perspectives en sciences sociales

Revue internationale de systémique complexe et d'études relationnelles

\title{
Le territoire, un système socio-patrimonial décrypté par le modèle de l'Acteur en 4 Dimensions
}

\section{Léa Sébastien}

Volume 10, numéro 1, novembre 2014

Sur le thème du territoire

URI : https://id.erudit.org/iderudit/1028442ar

DOI : https://doi.org/10.7202/1028442ar

Aller au sommaire du numéro

\section{Éditeur(s)}

Prise de parole

ISSN

1712-8307 (imprimé)

1918-7475 (numérique)

Découvrir la revue

\section{Citer cet article}

Sébastien, L. (2014). Le territoire, un système socio-patrimonial décrypté par le modèle de l'Acteur en 4 Dimensions. Nouvelles perspectives en sciences sociales, 10(1), 283-329. https://doi.org/10.7202/1028442ar
Résumé de l'article

Le territoire, entité relationnelle complexe, met en avant la dualité homme-nature en mêlant morphologie de l'espace et subjectivité du regard passé, présent et futur. Une notion polysémique donc, qui pour certains auteurs ne fait plus sens. Plutôt que d'abandonner la notion de territoire, nous proposons au contraire d'accepter la mouvance du concept et d'approfondir l'analyse de cette complexité territoriale. Pour ce faire, cet article expose deux thèses complémentaires. D'un point de vue théorique, nous positionnons le territoire en tant que système socio-patrimonial, fruit des relations sociales et des relations au patrimoine (naturel et culturel). Le territoire serait alors un noeud interactionnel entre ce que nous appelons rapport social et rapport patrimonial. D'un point de vue pratique, nous proposons d'opérationnaliser cette définition au-travers d'un modèle de diagnostic territorial intitulé l'Acteur en 4 Dimensions (A4D) qui vise l'analyse du jeu d'acteurs par l'étude des liens entre humains et liens au non humain. L'A4D permet de constituer ce que nous nommons des empreintes territoriales propres à chaque acteur, une radiographie synchronique des liens sociaux et patrimoniaux sur un territoire. Ce modèle conceptuel d'analyse est vecteur d'informations sur le jeu d'acteurs et de dialogue entre parties intéressées. Il s'adresse alors aux chercheurs, porteurs de projets, médiateurs ainsi qu'aux acteurs territoriaux eux-mêmes. Nos résultats montrent que l'étude des liens sociaux ne peut se faire indépendamment du contexte dans lesquels ils sont ancrés et inversement, que les liens homme-nature existent par le biais de relations sociales. Des objets qui font du lien ; des liens qui font des objets : une problématique à approfondir pour décrypter ce qu'est le territoire au-travers d'une analyse sur les attaches entre nature et culture. 


\title{
Le territoire, un système socio-patrimonial décrypté par le modèle de l'Acteur en 4 Dimensions
}

\author{
LÉa Sébastien \\ CNRS GEODE, Université Toulouse II
}

\section{Introduction}

Comment définir un territoire? Est-il espace : lieu de vie, lieu de mémoire, lieu de pratiques? Est-il patrimoine, héritage légué par les générations passées, que nous devons transmettre aux générations futures? Ou est-il tout cela en même temps, pour des acteurs qui ne doivent pas nécessairement partager des visions pour vivre ensemble? Dans tous les cas, la notion de territoire mêle éléments objectifs et subjectifs, associe ce qui a trait à l'humain à ce qui a trait à l'environnement.

Il s'agit en effet d'allier les deux pour comprendre un territoire. Pourtant les sciences sociales, à propos de sujets comme le développement local ou la gouvernance territoriale, cherchent encore à relier l'état de l'environnement d'un côté à l'organisation sociale de l'autre, sans en faire une analyse systémique. On s'intéresse en effet le plus souvent à l'acteur d'un système en tant qu'acteur social uniquement ${ }^{1}$, et pour comprendre le fonction-

$\overline{1}$ Marcel Jollivet et Alfredo Pena-Vega, «Relier les connaissances, transversalité, interdisciplinarité», Nature, Sciences et sociétés, vol. 10, n 1, 2002, p. 78-95. 
nement d'un territoire, on privilégiera l'étude des seuls rapports sociaux, qu'on tentera de relier avec l'état de l'environnement dans un second temps. La sociologie, en particulier, a été amenée à trop autonomiser son objet d'étude qui s'est retrouvé réduit à l'étude des seuls liens sociaux entre humains contemporains ${ }^{2}$. De même, en géographie, les approches isolent ce qui relève de l'organisation de l'espace et ce qui relève des acteurs ${ }^{3}$. Le territoire des géographes est en effet réducteur par son lien à la raison cartographique, formatant l'appréhension de l'espace et limitant l'analyse de la nature mobile des rapports sociaux à l'espace 4 . Par une entrée transdisciplinaire, il apparaît aujourd'hui nécessaire de s'intéresser aux rapports entre individu et nature, entre société et environnement physique, et finalement entre culture et nature 5 .

Nous proposons de redéfinir le territoire comme système socio-patrimonial, c'est-à-dire comme les interrelations entre rapports sociaux (liens entre acteurs) et ce que nous nommons rapports patrimoniaux (liens à l'espace et au temps). Afin de décrypter cette complexité territoriale, nous avons élaboré le modèle de l'Acteur en 4 dimensions (A4D) permettant un diagnostic du jeu d'acteurs territorial en identifiant et analysant les interactions entre rapport social et rapport patrimonial. Au travers de l'A4D, le territoire est donc à la fois objectivé par le

$\overline{2}$ André Micoud, «Entre Loire et Rhône, ou comment des objets naturels peuvent faire du lien", dans André Micoud et Michel Péroni (dir.), Ce qui nous relie, La Tour-d'Aigues, Éditions de l'Aube, 2000, p. 227-239.

3 Alexandre Moine, "Le territoire comme un système complexe : un concept opératoire pour l'aménagement et la géographie», L'espace géographique, vol. 2, tome 35, 2006, p. 115-132.

4 Frédéric Giraut, "Conceptualiser le territoire», Historiens et géographes, $\mathrm{n}^{\circ}$ 403, 2008, p. 57-68; Denis Retaillé, "L'espace mobile», dans Benoît Antheaume et Frédéric Giraut (dir.), Le territoire est mort. Vive les territoires!, Paris, Éditions de l'IRD, 2005, p. 175-201; Nadine Cattan «Gendering Mobility: Insights into the Construction of Spatial Concepts ", dans Tanu Prya Uteng et Tim Cresswell (dir.), Gendered Mobilities, Aldershot, Ashgate, 2008, p. 83-98.

5 Philippe Leroy, «La sociologie de l'environnement en Europe : évolution, champ d'action et ambivalences", Natures, Sciences et Sociétés, vol. 9, n ${ }^{\circ}$, 2001, p. 29-39. 
chercheur (lequel créé l'empreinte territoriale), vécu par l'habitant (analyse des représentations territoriales) et pratiqué par les acteurs locaux autour de projets (rôle du modèle dans une négociation territoriale). C'est par l'imbrication de ces trois approches que le modèle prend tout son sens.

Nous verrons dans une première partie le territoire en tant qu'entité relationnelle complexe que nous définissons comme système socio-patrimonial. Le modèle de l'A4D est présenté en deuxième partie, suivi des résultats généraux tirés de l'analyse des empreintes territoriales. La dernière partie discute des différents apports d'une telle approche et des questionnements qu'elle suscite à propos des relations homme-nature sur un territoire.

\section{I - Le territoire : une entité relationnelle complexe vue comme un système socio-patrimonial}

Le territoire est inscrit à l'agenda de nombreux champs disciplinaires, incluant le droit, les sciences politiques, l'économie et, évidemment, la géographie, qui en fait un concept central de sa discipline, lui permettant de situer l'acteur dans son espace. Les définitions de territoire ponctuent la recherche du XX' $\mathrm{X}^{\mathrm{e}}$ siècle jusqu'à aujourd'hui, avec comme point commun la mise en avant de la dualité homme et nature. Le territoire est une entité relationnelle complexe et peut être rapproché de nombreuses notions (espace, lieu, patrimoine, paysage). Nous proposons de traiter le territoire comme un système socio-patrimonial, alliage d'un patrimoine (rapport à l'espace et au temps) et de relations sociales.

1. Entre sujet et objet, un territoire pluridimensionnel

Emprunté au latin classique territorium, le mot "territoire» est défini par le Robert comme une «étendue de la surface terrestre sur laquelle vit un groupe humain». Au XIII e siècle, le latin chrétien lui donne le sens "de pays, paysage»; puis le territoire désigne une «étendue de terrain sur laquelle est établie une collectivité, spécialement qui relève d'une juridiction» $\left(\mathrm{XVII} \mathrm{e}^{\mathrm{e}}\right)$, «de l'autorité de l'État" (XVIII'). Le terme territorial, défini au $\mathrm{XVIII}^{e}$ par Montesquieu, est «ce qui concerne un territoire, du 
territoire». Il prend au XIX ${ }^{\mathrm{e}}$ un sens juridique et concerne «la défense du territoire national», selon Balzac qui l'utilise comme rural, agricole. Au même moment, l'éthologie définit le territoire comme l'espace qu'un animal délimite et s'approprie dans la mesure où il en interdit ou tente d'en interdire l'accès à certains animaux, en particulier ceux de sa propre espèce. Le territoire des animaux ne correspond pas à une portion bien délimitée de terre : il est mobile dans son tracé, variable selon les saisons mais approprié par ceux-ci. Par métaphore, il signifie «domaine, en parlant de ce qu'une personne considère comme sien ${ }^{6} »$. L'éthologie a largement participé à la diffusion puis l'analyse de la notion de territoire, en philosophie, anthropologie ou sociologie.

Dans le domaine du droit, on s'interroge sur un ordre juridique spatial, libéré de tout ancrage territorial. Les économistes parlent de développement endogène et local en lien avec le territoire. En sociologie, on évoque l'État territorial pour qualifier les nouvelles formes d'institutionnalisation de l'action collective et on présente la territorialité comme guide de l'action publique. Des ethnologues montrent que, pour entrer en politique, chaque société génère ses critères d'éligibilité territoriale. Le pouvoir local devient ainsi producteur et manipulateur de territorialité. La géopolitique, également, aborde le territoire comme espace de l'exercice de la souveraineté. Pour l'anthropologue Godelier, le territoire est «la portion de nature et d'espace qu'une société revendique comme le lieu où ses membres trouveront en permanence les conditions et les moyens matériels de leur existence ${ }^{7}$ ». Autrement dit, le territoire représente un espace sur lequel une société déterminée revendique et garantit à ses membres des droits stables d'accès, de contrôle et d'usage portant sur tout ou partie des ressources qui s'y trouvent et qu'elle est désireuse et capable d'exploiter. S'approprier collectivement un territoire, c'est se partager la maîtrise des conditions de reproduction de la vie des hommes, la leur propre, comme celle des ressources dont

\footnotetext{
6 Alain Rey, Le Robert : dictionnaire historique de la langue française, tomes 1, 2 et 3, Paris, Dictionnaire Le Robert, 1998.

7 Maurice Godelier, L'idéal et le matériel, Paris, Fayard, 1984, p. 78.
} 
ils dépendent. Il est possible alors d'envisager le territoire comme un pays (appropriation pour une production économique), un lieu (appropriation à des fins de commémoration culturelle) ou comme un paysage (appropriation d'un lieu en termes de singularité et d'expérience : souvenir, refuge, liberté). Dès lors, un territoire possède des valeurs emblématiques différentes selon les acteurs.

La multiplication des sens donnés au territoire induit une «surchauffe» du concept, qui plus est sans traduction satisfaisante anglo-saxonne. Dans le dictionnaire de Lévy et Lussault, huit définitions du territoire sont présentées ${ }^{8}$. Or une notion polysémique se vide de sens. La notion de territoire est-elle encore utile, $s^{\prime}$ interrogent Pecqueur ${ }^{9}$ ou Faure ${ }^{10}$ ? Certes le territoire recouvre une complexité difficile à saisir; il s'agit de comprendre la réalité qui nous entoure. Si la notion prend de plus en plus de place dans les débats contemporains, c'est que cette place est libre et qu'il convient non pas d'abandonner la notion de territoire mais d'accepter la mouvance du concept et, au contraire, d'approfondir l'analyse de cette complexité territoriale. Selon Giraut, «la complexité territoriale n'est ni une calamité ni un bienfait, c'est une expression spatiale des mutations sociales et des différentes facettes de la post-modernitén ${ }^{11} »$ qu'il convient de prendre en compte.

On peut présenter un territoire simplement comme un espace de vie, où coexistent ressources naturelles et culturelles. Dans ce contexte, est-il possible de définir les limites d'un territoire, et, si oui, de quelles manières? La réponse peut être simple si l'on se réfere à un découpage administratif, ou plus complexe si l'on se

$8 \quad$ Jacques Lévy et Michel Lussault, Dictionnaire de la géographie et de l'espace des sociétés, Paris, Belin, 2003.

9 Bernard Pecqueur, «De l'exténuation à la sublimation : la notion de territoire est-elle encore utile?», Géographie, économie, société, vol. 11, n 1, 2009, p. 55-62.

10 Alain Faure, «Territoires/territorialisation», dans Laurie Boussaget, Sophie Jaquot et Pauline Ravinet (dir.), Dictionnaire des politiques publiques, Paris, Presses de Sciences Po, 2004.

11 Frédéric Giraut, "Conceptualiser le territoire», Historiens et géographes, $n^{\circ} 403,2008$, p. 62. 
réfère à l'appréciation des groupes humains qui l'habitent ou de la répartition du vivant biologique. Un territoire est une entité fortement pluridimensionnelle qu'il est nécessaire d'aborder sous différents angles en englobant approches objectives et subjectives. Dans cette optique, il semble essentiel d'appréhender un territoire comme une entité relationnelle complexe constituée de trois dimensions indissociables : physique, biologique et humaine. Indissociables, oui, mais tout en gardant à l'esprit qu'un territoire est avant tout un complexe multi-acteurs où pratiques, représentations et savoirs s'entremêlent. Il peut être patrimoine, paysage, lieu, espace naturel, anthroposystème : il est co-évolution d'écosystèmes et de sociosystèmes. Appréhender un territoire nous place donc devant l'antithèse classique sujet-objet; en ce sens, un territoire ne peut être réduit ni à ses limites objectives, ni à ses limites subjectives.

\section{Le territoire... un espace?}

S'interroger sur la notion de territoire, c'est entrer dans le débat de la géographie sociale, laquelle tente d'explorer les interrelations qui existent entre rapports sociaux et rapports spatiaux, et plus généralement entre société et espace. Selon Di Méo, «le territoire témoigne d'une appropriation économique, idéologique et politique de l'espace par des groupes qui se donnent une représentation particulière d'eux-mêmes, de leur histoire, de leur singularité ${ }^{12}$ ». L'appropriation d'un espace par les acteurs devient le certificat de naissance du territoire ${ }^{13}$. Selon Benko, le territoire est l'espace qu'un groupe s'est approprié, pour ses besoins ${ }^{14}$.

Le territoire est espace vécu, espace approprié, espace aménagé, espace social. C'est en prenant en compte la notion de représentation dans l'approche géographique que l'espace peut être appréhendé en termes de sens, et considéré comme une donnée subjective.

\footnotetext{
12 Guy Di Méo, L’homme, la société, l'espace, Paris, Anthropos, 1990, p. 66.

13 Bernard Pecqueur, Le développement local, $2^{\mathrm{e}}$ édition, Dijon, Syros, 2000.

14 Georges Benko, "Territoires et sciences sociales», dans Xabier Itçaina, Jacques Palard et Sébastien Ségas (dir.), Régimes territoriaux et développement économique, Rennes, Presses universitaires de Rennes, 2007, p. 105-112.
} 
Le territoire peut être assimilé à une entité géographique suffisamment présente dans le sens commun pour faire l'objet de représentations collectives. L'espace représenté reconstruit l'espace de vie et le dépasse, brise les frontières pour le hisser jusqu'aux sphères de l'imaginaire, du rêve, de la mémoire et du concept ${ }^{15}$.

Le territoire présente une double nature, à la fois matérielle (l'espace géographique) et symbolique ou idéelle (systèmes de représentations) ${ }^{16}$. Travailler sur les représentations territoriales, c'est cerner toute la subjectivité qui relie l'individu à l'espace pour tenter d'appréhender la signification des lieux pour les hommes. Les rapports de l'homme à l'espace sont de deux ordres : 1) une géographicité de l'homme, c'est-à-dire un lien profond et individuel entre l'homme et le sol ${ }^{17}$ et 2) un rapport collectif d'une société à un espace construit, produit et chargé de sens par des pratiques de tous ordres. Chaque société produit alors des territoires, des espaces marqués par les pratiques, les représentations et les vécus humains à un moment de l'histoire.

Le territoire rejoint également l'idée d'espace au-travers de la notion de géosystème. Concept unificateur et intégrateur, le géosystème inclut l'écosystème en prenant en compte non seulement les interactions entre les systèmes vivants et leur environnement, mais, plus largement, les interrelations entre le milieu biophysique et les activités des sociétés humaines ${ }^{18}$. Ainsi le géosystème s'inscrit dans un espace et un temporalité, celle-ci incluant aussi bien le pas du temps historique que le temps actuel. Le géosystème aborde la question essentielle de l'anthropisation d'un espace naturel, mais est parfois détrôné par d'autres notions comme celles d'agro-écosystème ou d'écocomplexe. Néanmoins, les nouvelles problématiques environnementales et territoriales, créant un contexte académique où les frontières disciplinaires tendent à s'estomper, pourraient bien redonner au concept de géosystème toute sa pertinence.

\footnotetext{
15 Guy Di Méo, op. cit., p. 87.

16 Alexandre Moine, op. cit.

17 Éric Dardel, L'homme et la terre, vol. 6, Paris, Comité des travaux historiques et scientifique, 1990.

18 Georges Bertrand, "Paysages et géographie physique globale», RGPSO, tome 39, fasc. 3, 1968, p. 249-272.
} 


\section{Le territoire... un patrimoine?}

Si, au XII ${ }^{\mathrm{e}}$ siècle, patrimoine désigne "des biens de familles, le trésor public (patrimonium populi) et les biens ecclésiastiques (patrimonium Crucifixi)», son sens premier englobe «l'ensemble des biens appartenant au pater familias». Le XVIII siècle lui donne le sens figuré et plaisant de génitoires. Actuellement, sa définition générale est la suivante : "ce qui est transmis à une personne, à une collectivité par les ancêtres, les générations précédentes». En sociologie, il s'agit des «biens matériels et intellectuels hérités par une communauté» et, en génétique, du génome. Pour les économistes, il s'agit d'un bien susceptible de conserver dans le futur des potentialités d'adaptation à des usages non prévisibles dans le présent ${ }^{19}$. Dans le domaine du droit, le patrimoine est l'objet d'une construction juridique et représente "l'héritage qui apparaît comme l'instrument légal, institutionnel, ou le véhicule social des données en question : biens, terres, constructions, objets ${ }^{20}$ ".

À cette conception traditionnelle du patrimoine rattaché à une personne qui en est titulaire, s'oppose la théorie germanique du patrimoine, lequel est considéré comme un but, une idée. Cette théorie se rapproche de la vision contemporaine de la nature : masse de biens affectée à un but déterminé et qui serait détachée de toute personne stricto sensu. Cette conception de la nature réactualise la notion de res communis du droit romain, qui trouve une transcription moderne dans la notion de patrimoine commun, parfois sublimée par celle de patrimoine commun de l'humanité. C'est à partir des années 1970 que les termes patrimoine naturel, patrimoine de l'humanité, patrimoine génétique entrent dans les textes de loi. Rappelons que l'eau est patrimoine commun de la nation depuis 1992. Cette vision permet d'introduire la notion de bien commun, dépassant les clivages sujet/objet et bien public / bien privé, et d'aborder le monde dans sa complexité,

19 Jean de Montgolfier, Le patrimoine du futur. Approche pour une gestion patrimoniale des ressources naturelles, Paris, Economica, 1987.

Alain Chastel, Le Patrimoine, dans Symposium les enjeux, Paris, Encyclopædia Universalis, 1988, p. 268. 
pour faire du patrimoine le produit d'une sélection de représentations sociales.

Dans La nature hors la loi, Ost propose la notion de patrimoine comme «institution complexe qui articule le sujet et l'objet, le privé et le public, le local et le global, le présent, le passé et le futur ${ }^{21}$ ", patrimoine commun capable d'assurer non seulement le futur de la planète mais aussi celui des sociétés dont le fondement éthique recouvre les perspectives intergénérationnelles et environnementales. Bien que les expériences des groupes sociaux se localisent en un territoire donné, elles se projettent dans une dynamique globale et s'inscrivent dans une dimension de temps passé - savoirs et modes de vie - et futur. Dans cette perspective, Ost pense le patrimoine comme «institution trans-temporelle» et «trans-locale» en tant que réservoir de possibilités. Les biens reçus du passé doivent être transmis au futur, idée qui remet en question le principe de propriété, puisque l'examen des liens entre générations souligne le fait que les individus et les groupes sont gardiens d'un processus d'accumulation de savoirs et de pratiques. "Le patrimoine fait appel à l'idée d'un héritage légué par les générations qui nous ont précédés et que nous devons transmettre intact aux générations qui nous suivent ${ }^{22} »$. Comme le souligne Pupin, le patrimoine est une notion hybride qui intègre des éléments clés :

- le titulaire du patrimoine : une communauté de personnes physiques et morales peut être un titulaire légitime pour la gestion d'un patrimoine ne faisant pas l'objet d'un droit de propriété. Reste à définir comment la désigner et quelles en sont les responsabilités.

- le commun : une gestion commune, à distinguer de la gestion privée (individuelle) et de la gestion collective (puissance publique), est le mode de gestion le plus approprié

$\overline{21}$ François Ost, La nature hors la loi. L'écologie à l'épreuve du droit, Paris, La découverte, 1995, p. 54.

22 Michel Prieur, Droits de l'environnement, Paris, Dalloz, 1984, p. 45. 
des éléments trans-appropriatifs ${ }^{23}$. Reste à étudier qui sont les parties prenantes de la gestion commune.

- le rapport de l'homme au temps: la notion de transmission implique un choix lors de la perpétuation trans-générationnelle (passé-présent-futur) dans un monde caractérisé par l'incertitude et l'irréversibilité. Reste à identifier les éléments à transmettre aux générations futures.

- l'identité : il ne s'agit pas seulement de transmettre des éléments, il s'agit aussi de perpétuer l'existence de groupes d'acteurs et leur identité dans le temps. Reste à déterminer quelles sont les identités sur un territoire.

- l'unité : les éléments-clés du patrimoine présentés ci avant doivent être appréhendés de manière à constituer une unité tant au niveau du territoire, des acteurs, que de leur identité pour établir une cohérence. Une difficulté réside dans le fait qu'une telle unité n'est pas immuable et ne reste pas immobile dans le temps. ${ }^{24}$

Ces questions ouvertes rejoignent certaines problématiques en sociologie que sont, par exemple, le concept de patrimonialisation $^{25}$ ou les sciences de l'action avec la démarche patrimoniale ${ }^{26}$. Définir un patrimoine doit se faire par la confrontation des acteurs à une réalité commune. Devenu une notion à plusieurs ramifications, le terme patrimoine renvoie fondamentalement à la transmission des biens entre différentes générations; il désigne une totalité de "biens gérés en bon père de famille ${ }^{27}$ ». La notion de patrimoine qui appartient à l'origine au vocabulaire juridique a été reprise par la sociologie, l'économie et l'écologie; à chacun

23 Éléments échappant aux régimes des propriétés (ex : milieux naturels). Vincent Pupin, Les approches patrimoniales au regard de la question de la prise en charge du monde, Paris, Thèse de doctorat, Agro-Paris Tech, 2008. André Micoud, op. cit.

26 Henry Ollagnon, «Stratégies patrimoniales pour un développement durable», dans Christian Barrère et al., Patrimoine, approches croisées, n 2, École doctorale des sciences juridiques, économiques et de gestion, 2005.

Introduite dans le code civil à propos de l'usufruit, la gestion en bon père de famille prend en compte l'avenir. Elle impose au gestionnaire de ne pas dégrader les biens gérés, de les conserver et si nécessaire de les réparer. 
son patrimoine donc, mais on retrouve toujours l'idée de transmission d'entités des générations passées aux générations futures.

4. Notre proposition : le territoire comme système socio-patrimonial

Dans cet article, nous proposons de définir le territoire comme un système socio-patrimonial, c'est-à-dire en y associant patrimoine et relations entre acteurs. Comme le dit Paquot, «le territoire n'est pas le fruit d'un relief ni d'une donnée climatique mais devient l'enjeu de pouvoirs concurrents et divergents, et trouve sa légitimité avec les représentations qu'il génère, tant symboliques, patrimoniales et imaginaires ${ }^{28}$ ". Selon Di Méo, patrimoine et territoire offrent d'étonnantes correspondances : ces deux notions ont en commun de donner du sens et de la valeur à des objets, de participer à l'émergence d'un espace commun dans lequel le groupe se reconnaît et autour duquel il se construit. La notion de patrimoine intègre deux notions centrales dans la définition du territoire : la relation à l'espace et la relation au temps ${ }^{29}$. Comme le souligne Guérin, «le patrimoine, parce qu'il se réfère aux héritages, crée la personnalité du territoire $^{30}$ ». De même, Jolivet évoque « un espace communautaire, à la fois fonctionnel et symbolique, où des pratiques et une mémoire collective construites dans la durée ont permis de définir un Nous différencié et un sentiment d'appartenance ${ }^{31}$ ». Le territoire est ainsi ancré dans le passé mais également dans le présent au travers des actions locales et le futur au travers des projets de territoire. "C'est le lieu de constitution d'une société historique et d'une possibilité de vivre ensemble ${ }^{32} »$.

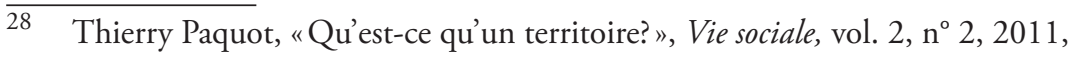
p. 26.

29 Guy Di Méo, Géographie sociale et territoire, Paris, Nathan, 1998.

30 Jean-Paul Guérin, "Patrimoine, patrimonialisation, enjeux géographiques», dans Jean-Marc Fournier (dir.), Faire la géographie sociale aujourd'hui, Caen, Presses universitaires de Caen, $\mathrm{n}^{\circ}$ 14, 2001, p. 44.

31 Marie-José Jolivet, "Logiques identitaires, logiques territoriales », Autrepart, $\mathrm{n}^{\circ} 14,2000$, p. 12.

32 Sylvie Biarez, "Pouvoirs et organisations locales : vers un nouveau paradigme politique", Sciences de la société, n 38, 1996, p. 43. 
Lien au temps, lien à l'espace donc et une troisième entrée pour définir un territoire est celle des acteurs qui font le territoire, entrée mentionnée mais rarement abordée de façon systémique. Il s'agit des «interrelations multiples qui lient ceux qui décident, perçoivent, s'opposent, s'allient, aménagent un territoire ${ }^{33}$ ». Ces acteurs sont concrets, repérables, ils font les territoires au travers des subtiles relations qu'ils entretiennent entre eux et auprès des objets patrimoniaux. Il s'agira d'analyser les rapports entre acteurs ainsi que les rapports de ceux-ci aux lieux qui les réunissent. Comme le dit Paasi "territories are made, given meanings, and destroyed in social and individual action. Hence, they are typically contested and actively negotiated ${ }^{34}$ ". La mémoire se couple ainsi à l'action pour définir le territoire. En effet, les territoires sont des systèmes autonomes d'action collective évoluant en fonction de valeurs (représentations) et comportements (pratiques) localisés. Le territoire est alors défini par le temps, résultat de pratiques sociales, produit de l'imaginaire et de l'idéologie des groupes; il est tout autant support de nos pratiques quotidiennes que produit de la société, entre la réalité et sa représentation ${ }^{35}$. La notion de patrimoine fait référence au temps, et donc à la mémoire, et possède en même temps une assise spatiale, une référence géographique ${ }^{36}$. Le patrimoine, reliant espace et temps, construit du territoire dans le rapport social.

Par rapport à la proposition de Moine ${ }^{37}$, qui définit le territoire comme un système complexe associant un ensemble d'acteurs, d'une part, et l'espace géographique utilisé, d'autre part, nous exprimons une réserve à propos de la notion d'espace qui n'intègre pas suffisamment clairement la dimension temporelle, essentielle

$33 \quad$ Alexandre Moine, op. cit.

34 Aansi Paasi, "Region and Place: Regional Identity in Question", Progress in Human Geography, vol. 27, n 4, 2003, p. 482; traduction : "les territoires sont créés, définis et détruits par l'action individuelle et sociale. Ils sont ainsi systématiquement contestés et négociés". dynamique et de ses ressources", Revue d'économie régionale et urbaine, $\mathrm{n}^{\circ} 5$, 2006, p. 683-700.

37 Alexandre Moine, op. cit. 
pour caractériser un territoire. Notre proposition pour le territoire est bien celle d'un système complexe mais associant le complexe multi-acteurs au patrimoine (alliant rapport au temps et à l'espace). Il existe deux niveaux de relations, qui vont à la fois lier entre eux les objets territoriaux localisés et ces mêmes objets avec des systèmes de relations entre des acteurs. Nous nommons rapport patrimonial le lien aux objets et rapport social le lien aux acteurs.

Les éléments patrimoniaux, en marquant fortement l'espace social, construisent du territoire. Ainsi peut-on considérer le territoire comme objet résultant d'un construit d'acteurs (patrimoine) ainsi que des relations entre eux. C'est l'infinité de relations liant les acteurs entre eux et les acteurs aux objets de l'espace qui crée le territoire. Nous définissons alors le territoire comme un système socio-patrimonial, c'est-à-dire comme des interrelations entre rapports sociaux et patrimoniaux sur un espace.

Le territoire apparaît comme un construit socioculturel qui se maintient et se renouvelle au fil de l'histoire au travers d'une dynamique d'accumulation collective des savoir-faire (pratiques), savoir-être (représentations) et des savoirs (connaissances) vis-àvis des autres acteurs et de leur milieu ${ }^{38}$. Nous proposons alors d'analyser les interrelations entre rapports sociaux et rapports patrimoniaux pour saisir un territoire; autrement dit, c'est en identifiant les pratiques, représentations et savoirs des acteurs vis-à-vis du monde social ainsi que de leur patrimoine que nous serons à même de décrypter la complexité territoriale. La figure 1 schématise notre proposition d'étude du territoire dont les deux sous-systèmes sont :

Rapport social (pratiques, représentations, savoirs des acteurs vis-à-vis des autres);

Rapport patrimonial (pratiques, représentations, savoirs des acteurs vis-à-vis de l'espace et du temps).

38 Gérard Donnadieu, La systémique : penser et agir dans la complexité, RueilMalmaison, Liaisons, 2002. 
Figure 1 :

Explicitations du territoire comme d'un système d'interrelations entre rapports social et patrimonial

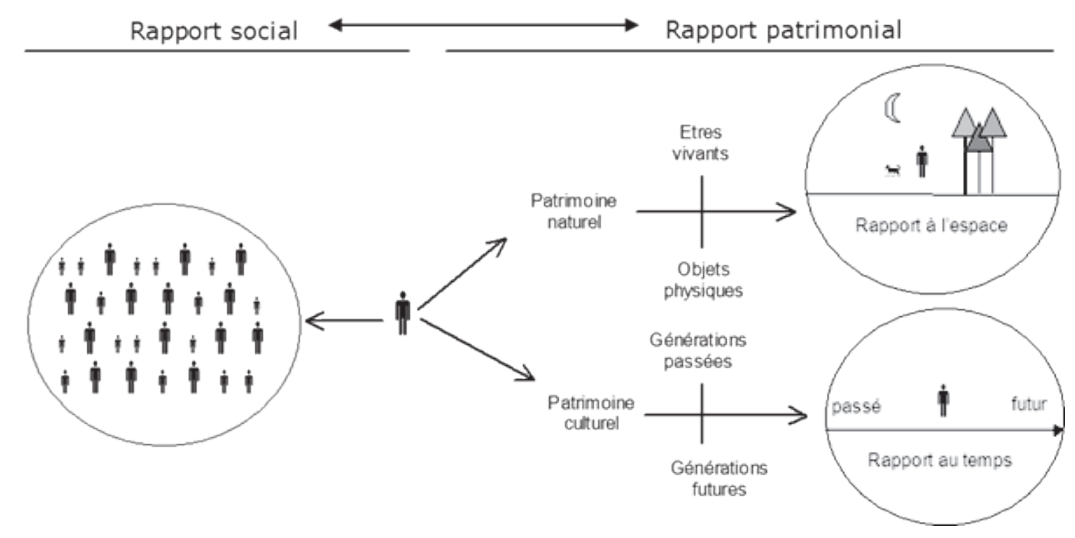

Les acteurs sont ici replacés au cœur du territoire et constitués en un écheveau complexe d'interrelations; la place centrale de l'acteur au sein du système est importante car il maintient la stabilité du système au sein duquel il agit. Notre définition de la complexité territoriale amène à analyser les jeux spatialisés des acteurs pour comprendre les processus qui guident l'évaluation du territoire. Pour ce faire, nous avons mis sur pied le modèle de l'Acteur en 4 dimensions reposant sur un diagnostic social et patrimonial du territoire.

\section{II- Présentation théorique du modèle del'Acteur en 4Dimensions}

Le modèle de l'A4D se donne comme objectif d'analyser un jeu d'acteurs territorial à la fois par les relations que tissent les acteurs entre eux (rapport social) ainsi que par les liens existants entre les acteurs et leur patrimoine naturel et culturel (rapport patrimonial). La théorie de l'acteur social sert de base pour l'étude des liens sociaux et positionne les acteurs dans leur coopération conflictuelle (tendance à la coopération et/ou au conflit avec les autres). De la même façon, c'est la notion de patrimonialisation qui inspire le rapport patrimonial des acteurs, afin de les positionner dans 
une cohabitation dominatrice (tendance à la cohabitation et/ou à la domination envers les éléments du territoire). L'A4D s'intéresse ensuite aux interactions entre rapport social et rapport patrimonial. Le modèle peut s'illustrer graphiquement au travers de l'empreinte territoriale, propre à chaque acteur. Celle-ci peut faire l'objet d'une représentation à 4 dimensions, 8 sous-dimensions ou 32 composantes. Elle représente une base d'informations sur le jeu d'acteurs et de dialogue entre parties intéressées.

\section{Lalliage des rapports social et patrimonial}

1.1. Le rapport social, un concept de sociologie des organisations Afin d'approcher la question des relations entre humains sur un territoire et de tenter de les évaluer, nous nous inspirons des sciences sociales, et notamment de la théorie de l'acteur social. Celle-ci est fondée sur la thèse suivante :

une organisation, phénomène sociologique, est toujours un construit social, qui existe et se transforme seulement si d'une part elle peut s'appuyer sur des jeux permettant d'intégrer les stratégies de ses participants et si d'autre part elle assure à ceux-ci l'autonomie d'agents libres et coopératifs [...] l'acteur est donc engagé dans un système d'action concret et doit découvrir, avec la marge de liberté dont il dispose, sa véritable responsabilité ${ }^{39}$.

Cette approche propose de donner du sens aux stratégies des acteurs et de connaître les jeux qui conditionnent leurs comportements. Elle vise donc à une meilleure compréhension du fonctionnement des organisations sociales.

Il s'agit d'une approche organisationnelle visant à découvrir les caractéristiques, la nature et les règles du jeu qui structurent les relations entre les acteurs concernés, celles-ci conditionnant leurs stratégies et, au final, les modes de régulation du système d'action. Le but est ici de produire des connaissances capables d'éclairer les pratiques des acteurs, non pas en formulant des lois générales, ni des recettes, mais en leur permettant de mieux se situer dans leur champ d'action et de mieux mesurer les

39 Michel Crozier et Erhard Friedberg, L'acteur et le système, Paris, Seuil, 1977, p. 27. 
contraintes et les interactions du jeu d'acteurs. Il est donc essentiel de prendre comme point de départ le vécu des acteurs.

Les principes de cette théorie permettent de construire des modèles d'analyses du jeu d'acteurs pour tenter de comprendre les phénomènes et la manière dont sont structurés (ou déstructurés) les rapports sociaux dont les acteurs sont parties prenantes et au travers desquels ils se constituent comme acteurs sociaux. L'acteur social est défini par la nature du rapport social dans lequel il est engagé. Cet acteur peut être individuel ou collectif. Dans tous les cas, un rapport social se présente comme une coopération conflictuelle d'acteurs qui coopèrent à une production mais qui entrent inévitablement en conflit en raison de leurs positions inégales dans la coopération ou, ce qui revient au même, de leur emprise inégale sur les enjeux de leur coopération ${ }^{40}$. Les acteurs d'un territoire sont donc interdépendants, ils coopèrent en ayant des intérêts divergents, parfois même contradictoires ${ }^{41}$. C'est ce que le rapport social tente de saisir.

Le rapport social est alors défini comme une relation de coopération et de conflit. Il s'agit de situer un acteur donné par rapport aux autres sur un plan de coopération conflictuelle, c'est-à-dire d'évaluer le potentiel de l'acteur à coopérer ou entrer en conflit avec les autres. Il est possible de mesurer ce potentiel à partir d'une série de composantes, elles-mêmes définies par plusieurs indicateurs. Au final, le rapport social peut faire l'objet d'une représentation graphique, grâce à un indice de coopération et de conflit, estimé pour chaque acteur (voir la figure 2). Une telle représentation peut paraître simpliste mais permet néanmoins de situer un acteur dans un espace défini par les deux dimensions du rapport social. On peut ensuite comparer les acteurs entre eux et mesurer leurs différences, notamment dans l'exploration de la nature et du rôle des acteurs faibles du territoire ${ }^{42}$.

40 Raymond Quivy et Luc van Campenhoudt, Manuel de recherche en sciences sociales, Paris, Dunod, 1995.

41 Ehrard Friedberg, Le pouvoir et la règle, Paris, Seuil, coll. "Sociologie», 1993.

42 Léa Sébastien, "Quand les acteurs faibles et absents s'immiscent dans la négociation environnementale», Territoire en mouvement, $\mathrm{n}^{\circ} 11,2011$, p. 66-81. 
Figure 2 :

Représentation graphique du rapport social

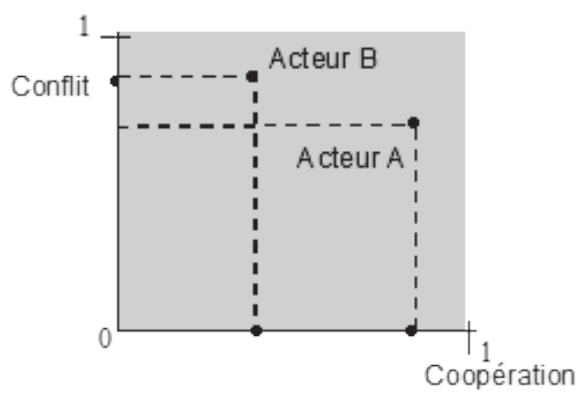

1.2. La patrimonialisation, une ouverture pour l'étude des liens homme-nature

a. Une gestion patrimoniale, pourquoi?

Comme évoqué en introduction, les sciences sociales s'intéressent à l'acteur d'un système en tant qu'acteur social uniquement et privilégient l'étude des seuls rapports sociaux pour comprendre le fonctionnement d'un territoire. L'homme moderne, considéré à travers la théorie de l'acteur social, a ainsi été construit, seulement social et seulement actuel. Deux aspects fondamentaux ont été dissociés de son étude et lui sont pourtant intimement et intrinsèquement reliés : la nature (milieu naturel et vivant biologique) et la tradition (générations futures et passées) ${ }^{43}$.

La montée de ces nouvelles aspirations avait été pressentie par le sociologue Edgar Morin en 1967; il écrivait dans sa monographie d'un village breton qu'il se manifestait dans certains îlots de la classe bourgeoise un "néo-archaïsme» traduisant l'inquiétude et l'insatisfaction face au modernisme et la recherche du «naturel, du primitif et du fondamental ${ }^{44} »$. Ces nouvelles aspirations se sont concrétisées en milieu rural par l'émergence simultanée de deux types d'attitudes : la revalorisation du passé et le regain

$43 \quad$ André Micoud, $o p$. cit.

44 Edgar Morin, Commune en France. La métamorphose de Plodémet, Paris, Fayard, 1967, p. 55. 
d'intérêt pour la nature. Ces comportements sont d'ailleurs intimement liés; dans les deux cas, il s'agit de préserver des témoignages du passé, une espèce devenue rare ou une ancienne pratique ${ }^{45}$. Michel de Certeau, dans un article qui tente d'analyser la pratique des historiens, estime que le passé, c'est d'abord «le moyen de représenter une différence [...] La figure du passé garde sa valeur première de représenter ce qui fait défaut [...] Un groupe, on le sait, ne peut exprimer ce qui manque encore que par une redistribution de son passé ${ }^{46}$ ".

Le concept de patrimonialisation est issu de la tradition anthropologique. Dans le domaine de l'eau par exemple, l'anthropologie fluviale considère tout autant un fleuve comme un patrimoine culturel porteur d'une mémoire, de légendes ou de pratiques identitaires. Le fleuve vivant est donc considéré de manière métaphorique, en tant qu'il est cela qui relie les humains qui vivent de lui, ou avec lui. Selon Micoud, la patrimonialisation est

un travail d'institution d'un nouveau cadre d'action. Ce travail [...] revient à prendre en charge la double question, proprement ontologique, de la différence et de l'identité : 1) de la différence entre les ensembles humains et ce qui n'est pas eux mais dont ils font partie (qui définit les patrimoines naturels) et 2) de l'identité dans le temps des ensembles humains (qui définit les patrimoines culturels). Soit, autrement dit, des liens avec les contemporains non humains [vivant biologique], et des liens avec les humains non contemporains [générations futures et passées $]^{47}$.

L'auteur définit la patrimonialisation comme «l'activité sociale consistant à faire quelque chose qui prend nom et valeur de patrimoine». Il n'y a donc pas de patrimoine en soi mais la construction d'une représentation à un moment donné par un acteur ou un groupe d'acteurs. Ainsi, le patrimoine ne serait pas seulement une reprise, une relecture du passé, mais aussi une

45 Georges Barouch, La décision en miettes. Système de pensée et d'action à l'ouvre dans les milieux naturels, Paris, L'Harmattan, coll. "Logiques sociales», 1989.

46 Michel de Certeau, "L'opération historique», dans Jacques Le Goff et Pierre Nora (dir.), Faire de l'Histoire, vol. 1, Nouveaux problèmes, Paris, Gallimard, NRF, 1974, p. 12.

47 André Micoud, op. cit., p. 43. 
façon d'envisager l'avenir sur le socle de l'identité. La patrimonialisation suppose que l'on distingue ce qui fait sens pour les acteurs, pour les "héritiers» (les populations locales) et non pour les personnes extérieures au territoire ${ }^{48}$. Une telle approche consiste, par exemple, à protéger des éléments du milieu naturel, en l'établissant comme patrimoine commun pris en charge par l'ensemble des acteurs concernés dans toutes ses composantes (économique, écologique, technico-administrative).

"La gestion patrimoniale consiste à partir d'une négociation, à choisir la façon dont les usages (ou les fonctions) des objets territoriaux seront agencés [...] la négociation est le centre de la gestion patrimoniale car si chaque acteur se sent responsable des caractéristiques du milieu alors il accepte et respecte les usages différents du $\operatorname{sien}^{49}$ ». Encore une fois, la négociation peut être fondée sur un compromis et accompagnée par des processus d'arbitrage (notamment par la puissance publique) ou de médiation.

Selon Serres, "par les contrats exclusivement sociaux, nous avons laissé le lien qui nous rattache au monde, celui qui relie le temps qui passe et coule au temps qu'il fait, celui qui met en relation les sciences sociales et les sciences de l'univers, le lien qui adresse notre langue aux choses muettes ${ }^{50}$ ». Autrement dit, il n'existe pas de collectif humain sans choses : les rapports entre les hommes passent par les choses, nos rapports aux choses passent par les hommes. Au contrat exclusivement social, il s'agit d'ajouter la passation d'un contrat naturel de symbiose et de réciprocité. Autrement dit, la nature conditionne la nature humaine, qui la conditionne à son tour. La nature se conduit comme un sujet.

$48 \quad$ Hugues François, op. cit.

49 Damien Puech, «La prise de conscience du caractère patrimonial de l'eau, origine de nouvelles perspectives de gestion de cet éléments naturel?», dans GIP Hydrosystèmes, Chantal Aspe et Patrick Point (dir.), L'eau en représentations : gestion des milieux aquatiques et représentations sociales, CEMAGREF, 1999 , p. 83.

50 Michel Serres, Le Contrat Naturel, Paris, Flammarion, 1992, p. 51. 
b. Une proposition : le rapport patrimonial

Nous avons voulu aborder l'acteur non seulement selon les relations qu'il entretient avec les autres (rapport social) mais aussi selon les liens qu'il a tissés avec différents objets qui composent le territoire, d'un point de vue naturel ou culturel, relation que nous qualifions de rapport patrimonial. Au pan social souvent étudié, il nous a semblé pertinent d'y accoler le pan patrimonial, par effet miroir au rapport social. Il s'agit ici de comprendre comment les humains contemporains (acteurs du territoire) perçoivent les acteurs absents ${ }^{51}$ afin d'évaluer ce qui relie aujourd'hui acteurs et territoire ${ }^{52}$. Comme pour le rapport social qui est identifié par deux dimensions, coopération et conflit, le rapport patrimonial sera lui aussi évalué selon deux dimensions : cohabitation et domination. Pour chaque acteur, nous choisissons de mesurer sa volonté à cohabiter (dimension cohabitation) avec son territoire ou à le dominer (dimension domination). Schématiquement, un acteur aura tendance à cohabiter avec son territoire s'il tient compte des générations futures et du vivant biologique dans ses projets; un acteur aura tendance à dominer son territoire s'il ne tient compte que de ses intérêts propres. Comme pour la théorie de l'acteur social, nous décidons de représenter la relation que nous appelons cohabitation dominatrice sur un graphique pour comparer les acteurs entre eux (voir la figure 3). Nous définissons alors le rapport patrimonial comme la caractérisation de la relation entre un acteur et sa nature, d'une part (milieu naturel, êtres vivants non humains), et sa culture, d'autre part (générations passées et futures).

\footnotetext{
51 Vivant biologique et générations futures.

52 Léa Sébastien, op. cit.
} 
Figure 3 :

Représentation graphique du rapport patrimonial

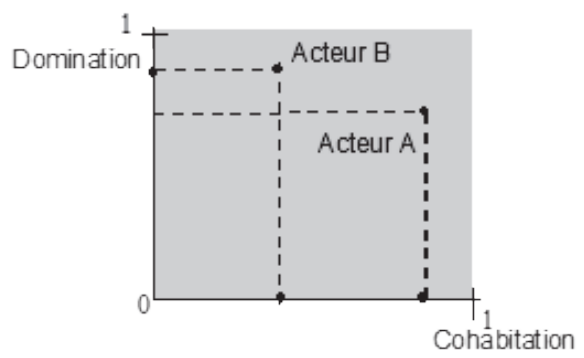

Ainsi, il n'est pas question de dissocier l'étude des relations entre humains des relations homme-territoire; rapport social et patrimonial seront analysés conjointement et dans la même logique. C'est en intégrant ces deux valeurs, rapport social et rapport patrimonial, que notre modèle conceptuel d'analyse prend tout son sens.

L'originalité de notre approche transdisciplinaire se situe dans la réunion de ces thèmes, en posant pour base que l'étude des liens sociaux ne peut se faire indépendamment du contexte dans lequel ils sont ancrés et, inversement, que les liens homme-nature existent par le biais de relations sociales. Autrement dit, l'A4D se propose de tenter d'établir un diagnostic territorial pertinent à partir de l'analyse des liens entre rapport patrimonial et rapport social, entre nature et culture.

\section{L'A4D : un outil affiné aboutissant à l'empreinte territoriale}

\subsection{La réflexivité du modèle}

Si la première étape de la construction du modèle fut l'élaboration du rapport patrimonial en vis-à-vis du rapport social, la seconde étape fut de scinder chaque dimension en deux sousdimensions. Afin de tenir compte du biais cognitif, de pondérer le discours de l'acteur et de rendre l'outil plus dynamique, des composantes tenant compte des perceptions ont été ajoutées au modèle et chaque dimension a été découpée en deux sous-dimensions. 
Ainsi, au niveau social, les sous-dimensions estime et critique sont renseignées par le discours de l'acteur (ce que dit l'acteur de luimême), estimé et critiqué sont renseignées par le discours des autres (ce que disent les autres de l'acteur). La dimension coopération comprend une sous-dimension estime, qui mesure la tendance d'un acteur à s'intéresser, à apprécier, à aller vers les autres, et une sous-dimension estimé qui mesure la tendance d'un acteur à intéresser et à attirer les autres, à en être apprécié. De la même façon, la dimension conflit comprend une sous-dimension critique qui mesure la tendance de l'acteur à se positionner contre les autres, et une sous-dimension critiqué qui mesure la tendance de l'acteur à générer l'animosité. Estime et critique marquent donc l'action de l'acteur sur le complexe, estimé et critiquél'action du complexe vers l'acteur. Autrement dit sont confrontées ici réactions de l'acteur et réactions des autres. C'est ce que nous appelons la réflexivité du modèle de l'A4D (voir la figure 4).

Figure 4 :

Explicitation des sous-dimensions

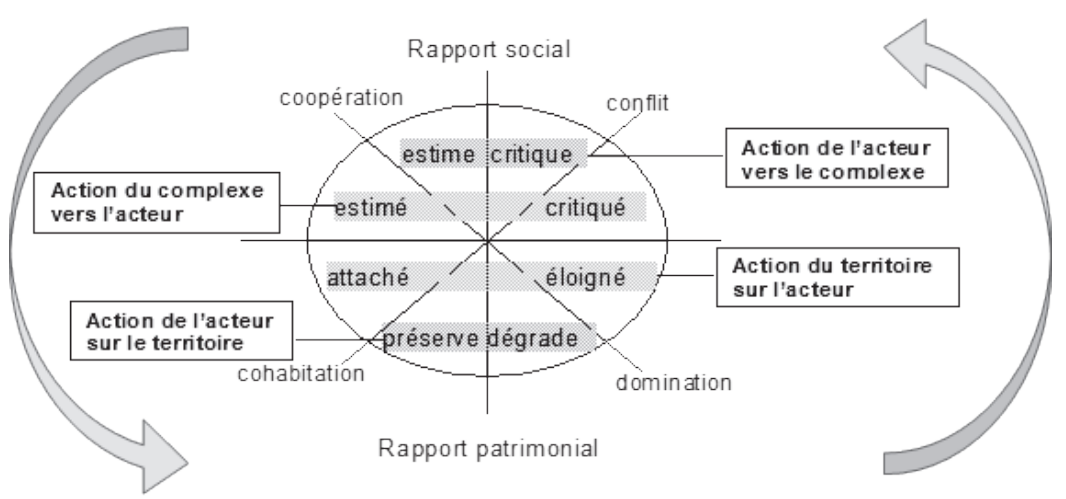

Le rapport patrimonial a été construit selon la même logique, en remplaçant néanmoins les autres acteurs par le territoire, c'est-à-dire en tentant de donner une voix au territoire. Comme le dit Moine $e^{53}$, les acteurs influencent le territoire tout autant que le territoire les influencent. Nous avons voulu exprimer cette

53 Alexandre Moine, op. cit. 
réflexivité au sein de notre modèle et souligner ici les interrelations entre acteur et territoire au sein du rapport patrimonial. Les objets territoriaux (naturels ou anthropiques) produisent des effets sur l'organisation des acteurs et vice versa, la localisation des uns et des autres impliquant un projet fondé sur un enchevêtrement de relations. Les objets du territoire entretiennent des relations avec les lieux ainsi qu'entre eux, cela par l'intermédiaire des acteurs. Selon Debarbieux, le territoire est un «agencement de ressources matérielles et symboliques capables de structurer les conditions pratiques de l'existence d'un collectif et d'informer en retour ce collectif sur son identité ${ }^{54} »$.

Ainsi, les deux sous-dimensions préserve et dégrade sont renseignées par le discours de l'acteur en question et celui des autres acteurs; attaché et éloigné, elles, tentent de témoigner de l'influence du territoire sur l'acteur. Autrement dit, on confronte ici réactions des acteurs et réactions du territoire. Selon Moles, la compréhension des relations que l'homme entretient avec l'espace qui l'entoure est régie par la loi proxémique issue de la psychologie de l'espace : «tout ce qui est proche (ici et maintenant) est pour moi plus important que ce qui est lointain (ailleurs, autrefois et plus tard) ${ }^{55}$ ». Dès lors, certains lieux du territoire, certaines entités peuvent faire l'objet de sentiments d'attachement ou d'appartenance. À l'inverse, d'autres lieux pourraient être l'objet de sentiments d'éloignement, de détachement. La dimension cohabitation comprend une sous-dimension préserve qui mesure la tendance d'un acteur à réaliser des actions de conservation, de préservation sur le territoire, et une sous-dimension attaché qui mesure l'ancrage de l'acteur dans le territoire. La dimension domination comprend une sous-dimension dégrade qui mesure le potentiel d'un acteur à exploiter, détruire, se protéger du territoire et une sous-dimension éloigné qui mesure la distance qui peut exister entre l'acteur et le territoire. Préserve et

\footnotetext{
54 Bernard Debarbieux, "Territoire», dans Jacques Lévy et Michel Lussault (dir.), Dictionnaire de la géographie et de l'espace des sociétés, Paris, Belin, 2003, p. 26.

55 Abraham Moles, "Vers une psycho-géographie», dans Antoine Bailly, Robert Ferras et Denise Pumain (dir.), Encyclopédie de géographie, Economica, 1995, p. 166.
} 
dégrade marquent l'action de l'acteur sur le territoire, attaché et éloigné l'action du territoire sur l'acteur. Une fois les sous-dimensions évaluées, il convient d'analyser leurs interrelations, par exemple dans quelle mesure un attachement à un fleuve (rapport patrimonial) peut engendre des actions coopératives ou conflictuelles (rapport social) ou, inversement, dans quelle mesure un conflit (rapport social) peut engendrer une dégradation environnementale (rapport patrimonial). L'A4D souhaite décrypter les rapports sociaux et patrimoniaux sur un territoire mais aussi leurs interactions. Des objets qui font du lien; des liens qui font des objets : telle est la complexité territoriale que l'A4D souhaite décrypter.

\subsection{Lempreinte territoriale, représentation graphique de l'A4D}

Au final, chaque groupe d'acteurs présente ce que nous appelons une empreinte territoriale ${ }^{56}$, empreinte qui résulte de la combinaison du rapport social et du rapport patrimonial. Chacune des 8 sous-dimensions est scindée en 4 composantes, soit 32 au total, qui sont chacune renseignée par une note allant de 0 à 5 (voir l'annexe pour l'exemple d'une sous-dimension). Regroupées par sous-dimension, cela donne graphiquement une note globale allant de 0 à 1 (voir la figure 5). L'ensemble de ces indicateurs découle tant d'un travail bibliographique important que de l'analyse des entretiens. La notation, inspirée de la méthode Mactor $^{57}$, peut être relative à des critères qualitatifs ou quantitatifs selon le cas et est attribuée en fonction des grilles d'analyse comparant les pratiques, représentations et savoirs des acteurs à propos des autres acteurs et des éléments composant leur territoire. L'empreinte territoriale, obtenue par report de ces notes sur un

\footnotetext{
56 Léa Sébastien, Humains et non-humains en pourparlers : l'Acteur en 4 Dimensions. Proposition théorique et méthodologique transdisciplinaire favorisant l'émancipation de nouvelles formes de gouvernances environnementales. Application au domaine de l'eau sur trois territoires : la Plaine du Forez, les pentes du Kilimandjaro et les Barthes de l'Adour, Thèse de doctorat, École des Mines de Saint-Étienne, 2006.

57 Michel Godet, Manuel de prospective stratégique, une indiscipline intellectuelle, Paris, Dunod, 2001.
} 
graphique, correspond à une radiographie des acteurs à un moment donné sur un territoire, explicitant leur rapport aux autres et aux patrimoines naturels et culturels (voir la figure 5). La force du modèle réside principalement dans sa capacité à passer d'entretiens semi-directifs à une objectivation du discours afin d'obtenir une représentation graphique des rapports sociaux et patrimoniaux d'un jeu d'acteurs territorial venant appuyer une analyse du discours. Le modèle a pu évoluer dans le temps, au fil des différentes expérimentations et des allers-retours théorie-terrain.

Rappelons que l'objectif du modèle est d'analyser conjointement rapport social et rapport patrimonial afin de proposer un diagnostic territorial pertinent, vecteur d'information sur le jeu d'acteurs et vecteur de dialogue grâce aux empreintes territoriales. Plus globalement, par notre approche transdisciplinaire, nous espérons faciliter la mise en place d'un processus de gouvernance pour une gestion intégrée et participative des ressources naturelles sur un territoire. En effet, l'A4D peut être mobilisé par un porteur de projet ou un médiateur territorial afin d'identifier les types de litiges et de créer du commun dans les sphères sociales comme patrimoniales. 
Figure 5:

Un exemple d'une empreinte territoriale

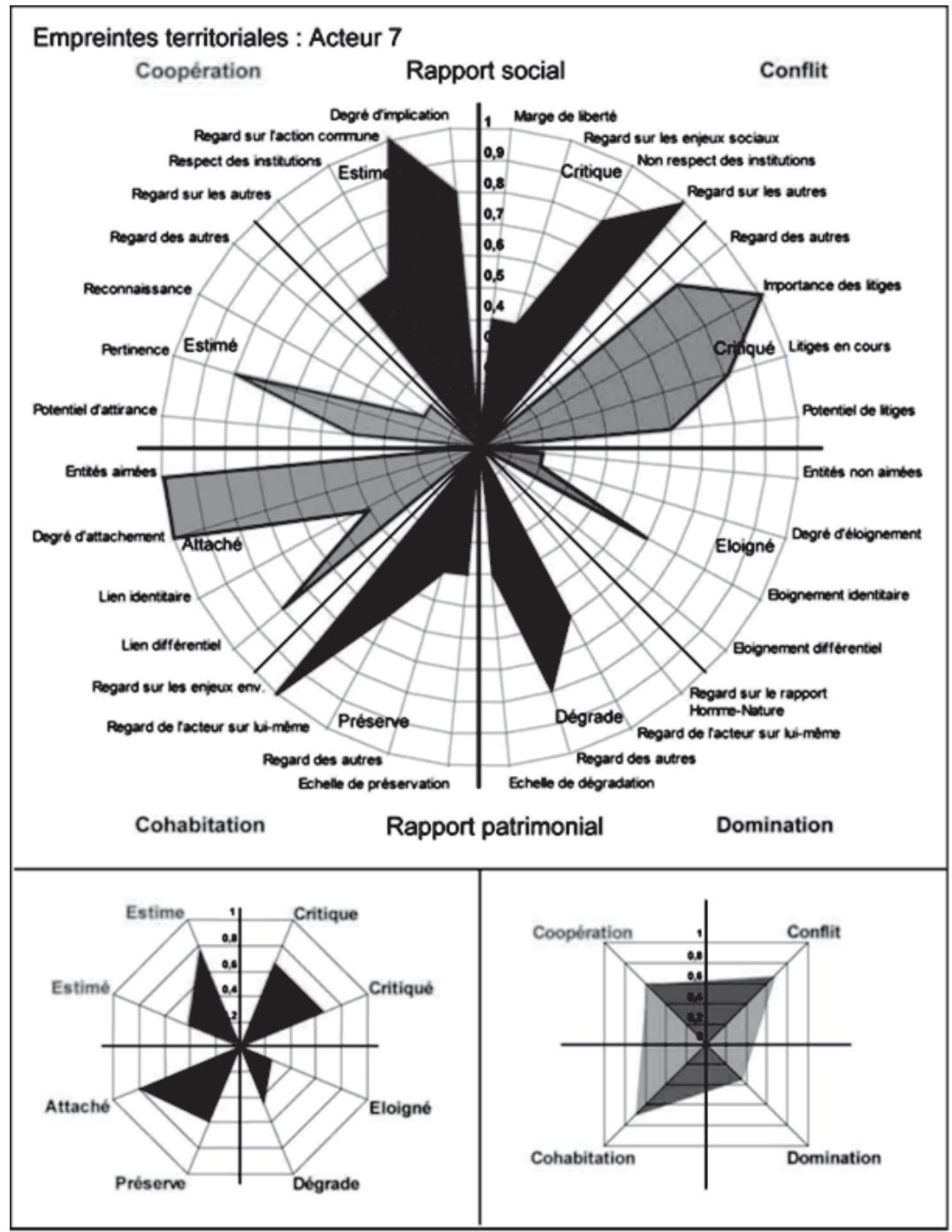

[en haut l'empreinte en 32 composantes, en bas à gauche l'empreinte en 8 sous-dimensions et en bas à droite l'empreinte en 4 dimensions.] 


\section{III - Application de l'A4D et résultats des empreintes territoriales}

Une fois le modèle pensé théoriquement, nous avons voulu tester sa robustesse en l'expérimentant sur plusieurs terrains radicalement différents : la Plaine du Forez, les pentes du Kilimandjaro, les Barthes de l'Adour, la vallée de la Neste. Nos résultats soulignent d'abord la diversité des liens qui existent entre sociétés et nature; ils montrent ensuite que rapports social et patrimonial sont interreliés sur chaque territoire et qu'il est possible d'envisager une typologie des acteurs selon leur empreinte territoriale.

1. Méthodologie pour un diagnostic territorial pertinent

Alliant éléments objectifs et subjectifs comme des entités relationnelles complexes, le territoire est un système socio-patrimonial qui fait le pont entre nature et culture. La cible de ce travail de recherche porte sur l'analyse des rapports sociaux et patrimoniaux sur quatre territoires radicalement différents, qui peuvent être considérés comme des laboratoires où, à partir d'analyses portant sur le rapport entre sociétés locales et ressources naturelles, on peut tenter d'élaborer des bilans et perspectives de réconciliation entre sociosphère et biosphère. L'éclatement des terrains et des perspectives ne signifie pas l'inexistence d'un objet scientifique commun, il en révèle seulement la complexité.

Notre méthodologie d'étude du jeu d'acteurs a ainsi été testée sur quatre territoires biophysiques appréhendés comme territoires vécus, milieux où vivent des ensembles humains, espaces constitués d'une mosaïque d'organisations politiques et administratives. Ce sont des formations socio-spatiales, des entités géographiques cohérentes, c'est-à-dire des espaces plus ou moins perceptibles et délimités mais toujours suffisamment présents, dans le sens commun, pour faire l'objet de représentations collectives. Ces formations socio-spatiales sont doublement objectivées par la superposition approximative des espaces vécus individuels, des 
entités écosystémiques et par l'effort organisationnel d'appareils politiques idéologiques ${ }^{58}$.

Il a paru pertinent de tester notre outil sur quatre territoires n'ayant a priori rien en commun, afin de tester à la fois la robustesse du modèle et sa flexibilité. Rappelons que notre travail ne s'intéresse pas à un projet territorial en particulier, mais bien à un territoire biophysique en lui-même (intégrant ou non des projets territoriaux). Un tel a priori permet de réfléchir à la manière dont notre outil peut poser les bases d'un projet, susciter du collectif par le biais d'une analyse du jeu d'acteurs sous l'angle socio-patrimonial.

Au niveau méthodologique, une fois les acteurs pertinents du territoire identifiés, leur avis est recueilli par l'intermédiaire d'entretiens semi-directifs organisés selon la méthode de stratégie patrimoniale $^{59}$. Chaque entretien a duré entre 1 et 2 heures et notre objectif était de tendre vers la diversité des acteurs rencontrés plutôt que l'exhaustivité.

$\mathrm{Au}$ total, lors de cette étude, 108 entretiens ont été réalisés entre 2005 et 2011 sur nos 4 terrains d'étude : 13 dans la Plaine du Forez, 58 sur les pentes du Kilimandjaro (dont 50 familles Chagga ${ }^{60}$ ), 14 dans les Barthes de l'Adour et 23 en vallée de Neste. Notre guide d'entretien vise à interroger les acteurs sur leurs connaissances, leurs pratiques, leurs préférences, mais aussi sur leurs perceptions personnelles de certains enjeux. L'idée sous-jacente est que, dans une négociation territoriale, les conflits de position et les divergences de représentation entrent en jeu de manière non négligeable ${ }^{61}$. Même si l'enjeu d'une négociation se trouve être un projet localisé, ce que les acteurs vont utiliser pour

\footnotetext{
58 Guy Di Méo, op. cit.

59 Henri Ollagnon, op. cit.

60 En général, les acteurs rencontrés représentent eux-mêmes ainsi qu’un groupe. Pour le cas où les individus sont sans représentant de groupe, nous avons multiplié les entretiens afin de recueillir la diversité des points de vue au sein d'une communauté particulière. C'est le cas de l'ethnie Chagga, vivant sur les pentes du Kilimandjaro. Nous avons ainsi interviewé 50 familles Chagga, 10 dans 5 villages différents.

61 Roger Bourque et Christian Thuderoz, Sociologie de la négociation, Paris, La Découverte et Syros, 2002.
} 
persuader l'autre est du domaine de l'affectivité, du subjectif et des valeurs morales ${ }^{62}$. Dans le but de faciliter un processus de négociation territoriale, il est ainsi majeur de connaître les visions du monde des acteurs, c'est-à-dire les représentations qu'ils se font personnellement de leur territoire : du milieu naturel et du complexe multi-acteurs. Nous proposons un classement des acteurs en 11 mondes distincts, qui appartiennent à 3 sphères générales (voir le tableau 1):

- sphère publique : Mondes des administrations, des collectivités territoriales, des établissements publics et de la recherche,

- sphère privée : Mondes de l'agriculture, de l'industrie, des propriétaires et des usagers

- sphère associative : Mondes de la chasse, de la pêche et de la protection de la nature

Tableau 1 :

Classement des acteurs rencontrés selon trois sphères et onze mondes

\begin{tabular}{|c|c|c|c|c|}
\hline Terrains & $\begin{array}{l}\text { Plaine du Forez } \\
(n=13)\end{array}$ & $\begin{array}{l}\text { Kilimandjaro } \\
(n=8+50)\end{array}$ & $\begin{array}{l}\text { Barthes de l'Adour } \\
(n=14)\end{array}$ & $\begin{array}{l}\text { Vallée de la Neste } \\
(n=23)\end{array}$ \\
\hline Sphères & \multicolumn{4}{|l|}{ Acteurs (\#) } \\
\hline publique & 6 & 3 & 6 & 9 \\
\hline privée & 3 & $2+50$ & 3 & 8 \\
\hline associative & 4 & 1 & 5 & 6 \\
\hline \multicolumn{5}{|l|}{ Mondes } \\
\hline Administrations & A9 & A5 & A8 & $\mathrm{A} 22, \mathrm{~A} 23$ \\
\hline Agriculture & $\mathrm{A} 8, \mathrm{~A} 10$ & A3 & $\mathrm{A} 1, \mathrm{~A} 10$ & $\mathrm{~A} 1, \mathrm{~A} 2, \mathrm{~A} 3, \mathrm{~A} 4$ \\
\hline Chasse & $A 6$ & & A14 & $A 21$ \\
\hline $\begin{array}{l}\text { Collectivités } \\
\text { territoriales }\end{array}$ & $\mathrm{A} 2, \mathrm{~A} 3, \mathrm{~A} 4$ & A6 & $\mathrm{A} 5, \mathrm{~A} 7, \mathrm{~A} 9$ & $\mathrm{~A} 19, \mathrm{~A} 20$ \\
\hline Etablissement public & A13 & A2 & A12, & A16, A17, A18 \\
\hline Industrie & $\mathrm{A} 7, \mathrm{~A} 12$ & A4 & & A15 \\
\hline Pêche & A1 & & A3 & A5 \\
\hline Propriétaires & & A8 & $\mathrm{A} 2, \mathrm{~A} 6$ & $\mathrm{~A} 6, \mathrm{~A} 7, \mathrm{~A} 8$ \\
\hline Protection nature & A11 & A1 & A11 & $\mathrm{A} 12, \mathrm{~A} 13, \mathrm{~A} 14$ \\
\hline Recherche & & A9 & A4 & A11 \\
\hline Usagers & A5 & A7 (50 familles) & A13 & $\mathrm{A} 9, \mathrm{~A} 10$ \\
\hline
\end{tabular}

$\overline{62}$ Olgierd Kuty, La négociation des valeurs : introduction à la sociologie, Bruxelles, De Boeck, coll. «Ouvertures sociologiques», 1998. 
Les Mondes définis et les titres associés aux acteurs représentent souvent des catégories employées par les enquêtés eux-mêmes. Non détaillées, elles présentent l'avantage de garantir l'anonymat des acteurs. En réalité, ces Mondes s'avèrent le plus souvent reliés les uns aux autres et certains acteurs s'inscrivent dans plusieurs catégories.

L'ensemble des discours ont été enregistrés (sauf sur le Kilimandjaro car la présence d'un traducteur autorisait la prise de notes) puis retranscris dans deux types de grilles d'analyse :

- une grille à trois catégories : savoirs (connaissances de type scientifique, institutionnel ou vernaculaire que mobilise l'acteur lors de l'entretien); représentations (savoir-être : perceptions, valeurs morales de l'acteur); pratiques (savoirfaire : actions, réalisations, projets de l'acteur ${ }^{63}$;

- une grille générale à 14 entrées : affinités, animosités, projet aimé, projet non aimé, avis concertation, avis préservation, problèmes $\mathrm{du}$ territoire, problèmes du groupe, entités aimées, entités non aimées, avis sur les autres, image de lui-même, atouts, acteurs à rencontrés.

Ces deux grilles d'analyse représentent notre base pour effectuer une analyse des discours et pour renseigner le modèle de l'A4D.

2. Analyse globale des empreintes territoriales

Ce qui frappe en premier lieu dans l'analyse des résultats, c'est que chacun des quatre terrains démontre des rapports social et patrimonial corrélés. L'analyse comparative des courbes des deux rapports montre qu' un fort rapport social va de pair avec un fort rapport patrimonial, et inversement, à une faible note en rapport social est associée une faible note en rapport patrimonial. Sur nos quatre terrains d'étude, un acteur impliqué sur son territoire l'est aussi socialement; un acteur distant du territoire l'est aussi vis-àvis du jeu d'acteurs. Notre démarche démontre un lien entre rapport social et rapport patrimonial, ce qui confirme nos hypothèses de départ.

$\overline{63}$ Gérard Donnadieu, op. cit. 
Cette démonstration rejoint les propos de nombreux auteurs présentant le territoire comme une entité pluri-dimensionnelle, co-évolution d'écosystèmes et de socio-systèmes, entre sujet et objet. Le territoire est envisagé comme une entité relationnelle complexe, fruit de l'évolution des représentations sociales, entre morphologie de l'environnement et psychologie du regard. Ainsi toute forme d'appropriation de la nature est-elle celle d'un rapport social. En définitive, "partout apparaît un lien intime entre la manière d'user de la nature et la manière d'user de l'homme ${ }^{64}$ ».

En allant un peu plus loin, remarquons que, sur chacun de nos quatre terrains, la dimension cohabitation est corrélée aux dimensions coopération et conflit, autrement dit avec le rapport social dans sa globalité et, inversement, la dimension domination est corrélée négativement avec les dimensions du rapport social. Il est à constater que, dans la Plaine du Forez, sur les pentes du Kilimandjaro, dans les Barthes de l'Adour et sur la vallée de la Neste, les acteurs qui présentent une forte tendance à la cohabitation démontrent un fort rapport social, de fortes notes à la fois dans la coopération et dans le conflit. Inversement, les acteurs qui sont ancrés dans la domination présentent de faibles notes dans le rapport social. Sur nos terrains, la domination territoriale est alors synonyme d'isolement social et la cohabitation traduit une implication active dans le jeu d'acteurs. Il est vrai que les dominateurs, souvent des acteurs puissants et autonomes, ne recherchent aucun partenaire, se montrent réticents à la concertation et vivent finalement bien leur isolement social. Au contraire, les préservateurs se disent souvent non entendus, n'hésitent pas à critiquer les autres, cherchent des alliances et sont à la fois estimés et critiqués pour leurs actions sur le territoire.

Autre point commun aux quatre terrains d'étude : la composante regard des autres des sous-dimensions estimé et préserve présente systématiquement une note faible, et la composante regard sur les autres des sous-dimensions critique et dégrade est toujours accentuée. Autrement dit, sur nos territoires, un acteur avoue très rarement apprécier les autres et reconnaître leurs actions de protection des

64 Maurice Godelier, op. cit., p. 46. 
milieux (regard des autres), mais ce même acteur n'hésite pas à critiquer fortement les autres et à pointer du doigt leurs actions de dégradation environnementale (regard sur les autres). Ainsi, les actions de protection de la nature passent totalement inaperçues, ne sont nullement reconnues par le complexe multi-acteurs tandis que les dégradations environnementales sont toujours mises en exergue, décriées par celui-ci.

Finalement, on note que, sur nos quatre territoires, les notes du rapport social dépassent toujours celles du rapport patrimonial. En observant ce résultat de plus près, il serait faux de dire que les relations sociales constituent une priorité par rapport aux liens patrimoniaux. Mais autant est-il courant de rencontrer des acteurs ayant de nombreux alliés et opposants, et s'inscrivant ainsi à la fois dans la coopération et le conflit, autant est-il rare de trouver de fortes notes à la fois dans la cohabitation et la domination. Cette observation concerne les actions territoriales et non les perceptions. En effet, certains acteurs sont à la fois éloignés et attachés. En revanche, un acteur s'engage dans de grandes actions de préservation ou est responsable de lourdes dégradations, mais il sera rarement présent sur les deux fronts.

Concernant la notion de territoire, il évoque pour les acteurs de nos terrains d'étude des représentations divergentes. Certains en parlent comme d'un simple espace, support d'activités économiques, d'autres comme d'un paysage à entretenir ou d'un patrimoine à conserver. Le territoire véhicule alors de nombreuses images et symboles. Définir un territoire vécu idéal commun devient dans ces conditions un objectif plus qu'ambitieux. On remarque trois sphères dans lesquelles se situent les acteurs rencontrés sur nos quatre territoires :

- la sphère technique concerne les acteurs qui avant toute chose mènent des actions de terrain, organisent des projets sur le territoire, sont spécialistes d'un domaine, sont confrontés aux réalités du territoire, privilégient l'action au discours;

- la sphère théorique concerne les acteurs qui appuient leur discours sur des connaissances institutionnelles et scientifiques, 
présentent des stratégies abstraites basées sur des logiques construites à partir de bases de données; ils sont rarement sur le terrain;

- la sphère affective concerne les acteurs qui se basent sur leur vécu personnel pour évoquer un sujet; ils n'hésitent pas à être subjectifs, à parler de sentiments et de souvenirs.

C'est par l'interpénétration de l'ensemble de ces pratiques de terrain, de ces connaissances diverses et de ces sentiments que peuvent éventuellement se co-construire un patrimoine commun, une conscience environnementale (centrée sur les savoirs), et même, éventuellement, une certaine sensibilité environnementale (centrée sur l'attachement).

\section{Spécificité des terrains d'étude}

Dans la Plaine du Forez, les empreintes territoriales individuelles font apparaître une diversité d'acteurs présents sur le territoire et soulignent ce que chacun peut apporter à une négociation environnementale. La synthèse globale dévoile un jeu d'acteurs marqué par les dimensions cohabitation et conflit. L'opposition affichée entre protection de la nature et protection du cadre de vie se traduit par un conflit ouvert entre conservationnistes et ruraux. Le regard sur le rapport homme-nature offre une large palette de perceptions au sein du jeu d'acteurs. Certains veulent une nature sanctuarisée, d'autres une nature entretenue; certains souhaitent une concertation large et ouverte, d'autres jugent la concertation inutile. Seuls quelques consensuels apparaissent entre les deux, présentant des empreintes mitigées. Un objet cristallise l'ensemble de ces divergences de représentations : le fleuve Loire. Évoqué systématiquement dans les discours, il rassemble, fait et défait du lien social. Quant à la nappe phréatique, il s'agit de l'entité oubliée des acteurs. Sur ce territoire, le rapport social semble prédominant : pour conserver un statut ou changer d'image, les acteurs sont prêts à modifier leurs pratiques environnementales.

Sur les pentes du Kilimandjaro, les acteurs sont ancrés sur un même territoire mais vivent dans des réalités sociales différentes. 
La synthèse globale montre un jeu d'acteurs dominé par le conflit, la plupart des acteurs étant à la fois critiques et critiqués. Si la forêt d'altitude fait l'unanimité, les moyens pour la préserver sont sources de litiges majeurs entre acteurs. Sur ce territoire, à l'opposé de la Plaine du Forez, c'est le rapport patrimonial qui semble prédominant, dans la dégradation comme la préservation, dans l'attachement comme l'éloignement : les acteurs sont accrochés à leur territoire et ne sont nullement prêts à modifier leurs pratiques, peu importe l'image qu'ils dégagent auprès des autres. Source de revenus importants pour les autorités par le biais du tourisme, le patrimoine naturel du toit de l'Afrique «ne doit pas» se dégrader. Et pourtant... Face à la dégradation environnementale indéniable, on pointe du doigt le paysan Chagga, pour ses pratiques destructrices. Des 50 familles Chagga rencontrées, notre étude montre qu'elles manifestent un attachement profond à leur environnement et montrent des savoirs riches sur l'évolution de leur territoire. Étant prêts à faire des concessions pour protéger le milieu naturel, les Chagga sont loin de représenter les bourreaux écologiques qu'on accuse.

Dans les Barthes de l'Adour, l'analyse des empreintes territoriales individuelles nous montre quelques irréductibles acteurs très attachés à leur territoire et une grosse majorité qui présente des empreintes mitigées, un peu dans toutes les dimensions, mais dans aucune en particulier. L'influence de ces acteurs flottants se ressent dans la synthèse globale qui souligne l'importance des notes dans la moyenne. Étonnamment, le fleuve Adour est peu abordé dans les discours, contrairement aux prairies pâturées qui représentent le fleuron des Barthes selon les acteurs, un fleuron que les acteurs institutionnels tentent doucement de protéger. Contrairement à nos autres terrains, il n'existe pas d'association de protection de la nature revendicative sur le territoire des Barthes de l'Adour. Si la conservation existe, elle a lieu dans la concertation et le compromis. Sur ce terrain, il semble que ni le rapport social, ni le rapport patrimonial ne prédomine; ces deux rapports étant relativement faibles. Sauf quelques projets 
institutionnels de restauration du patrimoine, il semble que les Barthes soient à la recherche d'une nouvelle identité.

Dans la vallée de la Neste, c'est le rapport patrimonial qui prédomine avec de fortes empreintes notamment dans les sousdimensions attachement et éloignement. Les acteurs se disent fortement attachés aux prairies de haute montagne qu'ils considèrent comme un patrimoine extraordinaire mais peu d'acteurs sont impliqués dans leur conservation. Les arbres, au contraire, sont vus comme des envahisseurs du territoire mais, là aussi, les acteurs sont rarement impliqués dans des pratiques en lien avec les ligneux. C'est donc un rapport patrimonial déconnecté de la réalité, mais empreint d'une diversité de savoirs et de représentations. Les agriculteurs sont ceux avec les plus fortes empreintes tant sociales que patrimoniales, à la fois estimés et critiqués par les autres et vus à la fois pour leurs actions de conservation et de dégradation environnementales. Les acteurs déplorent le manque de concertation territoriale ou de projet fédérateur pour la vallée qui se morcelle.

\section{Typologie des empreintes}

En compilant l'ensemble des résultats de l'Acteur en 4 Dimensions sur nos quatre territoires, il est possible d'envisager une typologie des acteurs, selon les formes répétitives des empreintes territoriales. En effet, on retrouve quatre grands types d'empreintes qui reviennent continuellement au cours des territoires étudiés. Nous présentons ci-après l'acteur de manière générale, puis spécifions si l'acteur se fait le porte-voix d'acteurs faibles (sous-représentés) et/ou le porte-parole d'acteurs absents (vivant biologique et générations futures) dans ses rapports sociaux et patrimoniaux ${ }^{65}$. Dans le cadre éventuel d'un projet de territoire, nous notons également sa position vis-à-vis des processus de concertation et conservation.

En premier lieu, on retrouve le groupe des consensuellement corrects, lesquels sont essentiellement représentés par des acteurs institutionnels et qui sont caractérisés par un fort potentiel de 
coopération et une tendance à la cohabitation (voir la figure 6). Identifiant de nombreux alliés et peu d'opposants, ces acteurs sont le plus souvent appréciés des autres et, se présentant comme consensuels, ils sont peu sujets au conflit. Voués à la coopération, ils rejoignent la théorie de la négociation gagnant-gagnant où satisfaire ses besoins est aussi important que satisfaire les besoins de l'autre. Peu présents sur le terrain, ces consensuels se font les porte-parole d'entités défendues au niveau national, voire global, dans les lois ou les grands programmes institutionnels. Ils sont favorables à une conservation synonyme d'équilibre entre activités humaines et respect des milieux naturels et tentent souvent d'organiser des plateformes de concertation qu'ils souhaitent ouvertes au plus grand nombre. Ils ne se font néanmoins portevoix d'aucun acteur faible en particulier et, même s'ils prônent la coopération, se retrouvent souvent en dehors des problématiques territoriales locales.

Figure 6 :

Empreinte territoriale type du «consensuellement correct»

\section{le cons ens uellement correct}

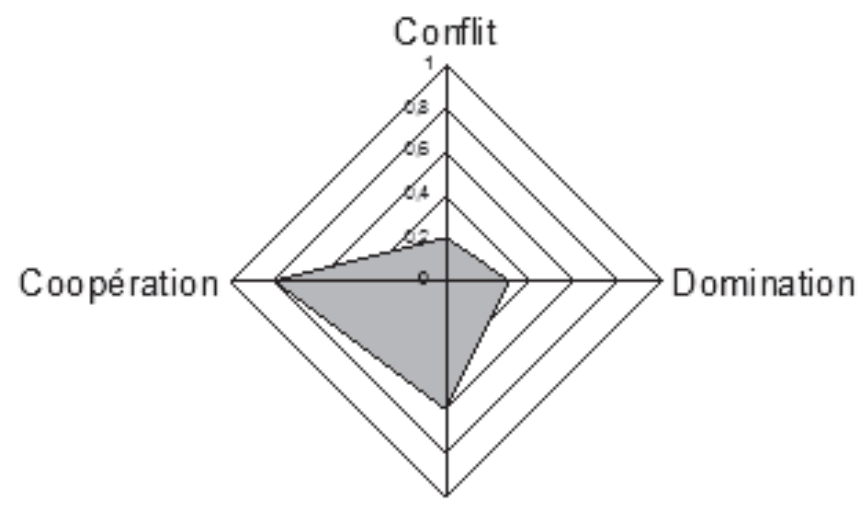

Cohabitation 
En second lieu, on retrouve sur nos trois territoires l'empreinte territoriale du protecteur conflictuel, caractérisé par une forte tendance à la cohabitation et au conflit, les deux autres dimensions étant très faiblement renseignées (voir la figure 7). Représentés par des associations et des usagers, ces acteurs apparaissent très attachés à plusieurs objets du territoire et engagés dans des actions de préservation. Ces conservationnistes montrent des positions tranchées en matière de protection de la nature qu'ils envisagent comme une sanctuarisation, devant être encadrée par la réglementation. Peu ouverts au compromis, ils n'hésitent pas à critiquer ouvertement les autres et sont impliqués dans de nombreux litiges, essentiellement des conflits de personne et de valeur morale. Ils vouent à la nature une valeur existentielle et s'ils se font les porte-parole de nombreuses entités du territoire, ils ne sont que rarement les porte-voix d'acteurs faibles.

Figure 7 :

Empreinte territoriale type du "protecteur conflictuel»

\section{le protecteur conflictuel}

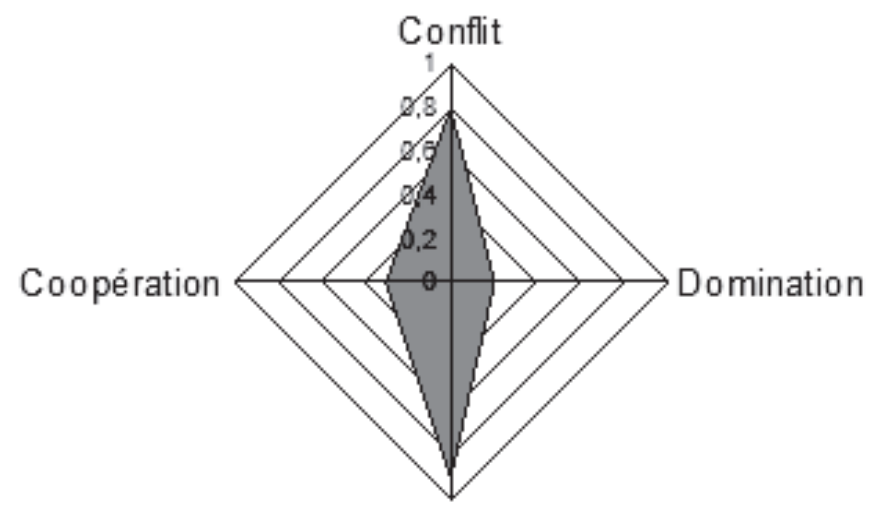

Cohabitation

Le groupe des petits cubiques est également très présent sur nos terrains d'étude. Ils se caractérisent par une empreinte territoriale équilibrée et de faible ampleur (voir la figure 8). En effet, ces 
acteurs ont comme principale caractéristique de s'inscrire équitablement dans chacune des 4 dimensions, avec des notes relativement faibles. Ces mitigés sont difficiles à cerner et à canaliser dans une négociation puisqu'ils présentent des potentiels, quoique modérés, à la fois de conflit, de coopération, de cohabitation et de domination. Ils sont souvent représentés par les élus et les scientifiques, ils savent identifier quelques alliés et opposants et sont engagés dans certaines actions de préservation comme de dégradation. Ils se font porte-parole d'objets reliés à leur activité et porte-voix de leurs clients (administrés, adhérents). Ils sont favorables à la conservation si elle ne remet pas en cause la rentabilité économique et à la concertation si elle est restreinte aux parties concernées. Un médiateur devra prendre garde aux différents indicateurs propres à l'acteur pour analyser plus en détail ce type d'empreinte cubique qui, par définition, ne se positionne clairement dans aucune dimension.

Figure 8 :

Empreinte territoriale type du "petit cubique»

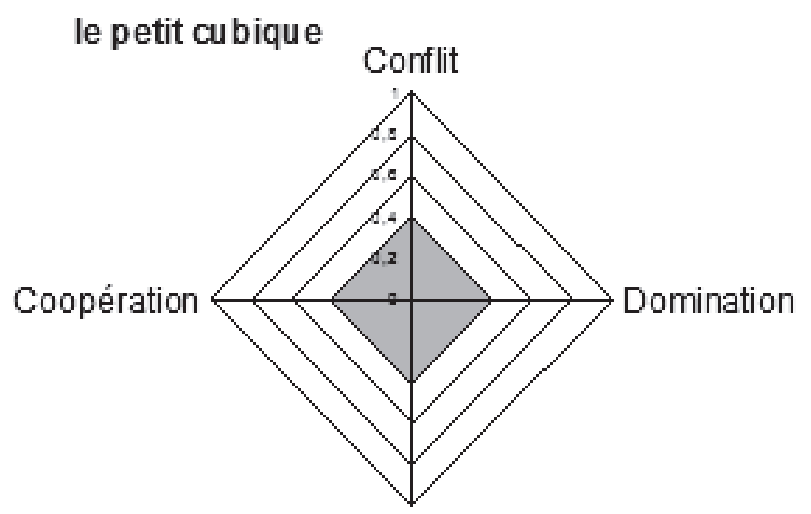

Cohabitation

Finalement, une empreinte territoriale rencontrée très fréquemment est celle des dominateurs distants, qui s'inscrivent nettement dans la domination, quelque peu dans le conflit et très 
faiblement dans les deux autres dimensions (voir la figure 9). Autonomes, influents et puissants, ces acteurs sont responsables d'actions de dégradation environnementale sur le territoire et s'avèrent imperméables aux critiques des autres. Représentés le plus souvent par les industriels, ces dominateurs semblent distants des problématiques territoriales et restent centrés sur leurs activités. Dès lors, ils se font rarement le porte-parole d'acteurs absents et le porte-voix d'acteurs faibles. Selon eux, la conservation ne doit pas freiner les activités économiques et la concertation doit être encadrée par un homme fort, un meneur, qui puisse trancher en fin de discussion (de préférence eux-mêmes).

Figure 9 :

Empreinte territoriale type du «dominateur distant»

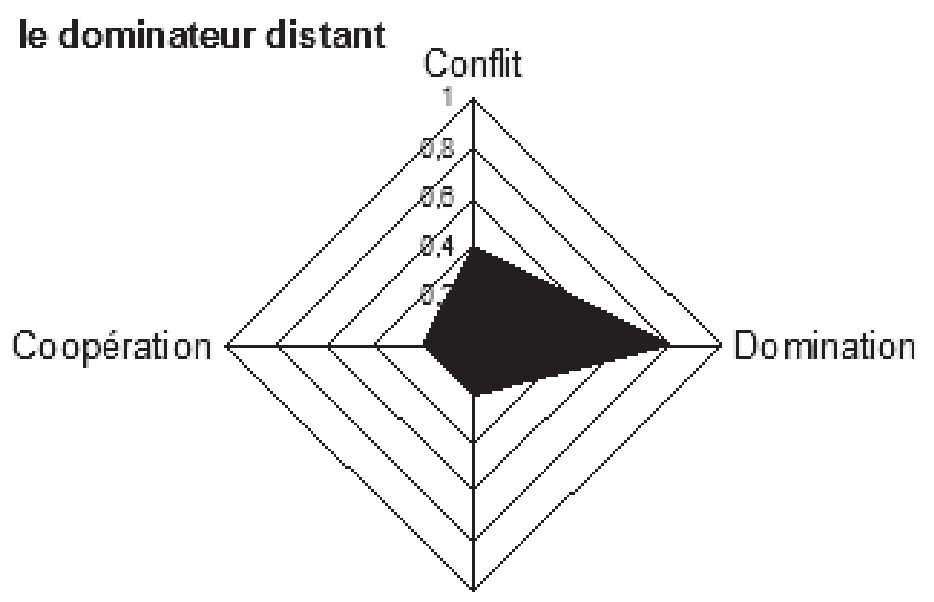

Cohabitation

\section{Les apports de l'A4D}

Alliant approches quantitatives et qualitatives, notre méthodologie se veut innovante tant aux niveaux théorique et scientifique qu'aux niveaux pratique et technique. Outre la sociologie qui sert de trame à l'A4D, notamment pour approcher les représentations sociales des acteurs, l'écologie nous aide à caractériser les écosys- 
tèmes du territoire, l'anthropologie appuie notre recherche du lien entre nature et culture, la prospective stratégique nous offre une base d'approche du jeu d'acteurs, la géographie propose des clés pour déceler le lien des acteurs à l'espace, les sciences politiques permettent de saisir la place des acteurs au sein des grands programmes institutionnels et les sciences économiques nous aiguillent sur les types de valeurs attribuées à la nature. Et l'instrument finalisé s'apparente dans sa forme à un modèle d'ingénierie sociale, mais où données quantitatives et qualitatives s'entremêlent. L'A4D se présente alors comme un modèle d'ingénierie sociale, fruit d'une réflexion transdisciplinaire et d'allers et retours entre théorie et terrain.

Parallèlement, la notion de rapport patrimonial enrichit la théorie de l'acteur social, puisqu'à l'étude des liens entre acteurs, est ajoutée l'analyse des liens de ce qui n'est pas eux, c'est-à-dire le vivant biologique et les générations futures. L'A4D peut aussi servir d'illustration ou d'application de terrain de la notion de patrimonialisation, qui s'intéresse aux patrimoines naturels et culturels des sociétés.

Un tel modèle fournit une vision globale d'un jeu d'acteurs sur un territoire, avec ses carences et ses points forts, et dévoile les convergences et divergences des pratiques, représentations et savoirs entre acteurs à propos du complexe multi-acteurs et du patrimoine territorial. L'outil peut alors servir pour un médiateur ou porteur de projet qui souhaiterait prendre connaissance d'un diagnostic territorial; l'A4D est alors vecteur d'informations. Qui plus est, la confrontation des acteurs à leurs propres empreintes territoriales crée des débats et peut servir de base pour une discussion, négociation ou concertation entre parties prenantes; l'A4D est alors vecteur de dialogue. Il est intéressant de noter qu'une empreinte territoriale est le fruit de la combinaison des discours de l'acteur lui-même et de ceux des autres. En effet, les indicateurs et composantes de l'A4D d'un acteur en particulier sont renseignés équitablement par les propos de l'acteur en question ainsi que par l'avis des autres sur ce même acteur; c'est ce que nous appelons la réflexivité du modèle. Cet apport 
méthodologique permet de pondérer les propos de l'acteur et de le positionner directement vis-à-vis du complexe. Il est à noter également que l'entretien mêle questions objectives et subjectives, faits et opinions, positionnements de l'organisme comme de l'individu. Notre méthodologie tente alors de saisir l'acteur dans sa globalité, c'est-à-dire en y intégrant son vécu, ses désirs et appréhensions, ses opinions et perceptions. On ne cherche pas uniquement à rencontrer un représentant de groupe, mais aussi une personne, pétrie d'histoires. Unir le personnel et le professionnel, l'objet et le sujet n'est certes pas le plus simple à analyser mais révèle la complexité et la richesse des rapports social et patrimonial sur un territoire.

Finalement, l'A4D peut alors prendre les allures d'un outil d'aide à l'action visant à faire accepter la diversité en se révélant un moyen de représentation de la diversité territoriale, une diversité qui témoigne de la coévolution au fil du temps des sociosystèmes et des écosystèmes.

\section{Conclusion}

Alberto Magnaghi nous dit que le territoire est une sorte de mille-feuille, chaque strate témoignant d'un épisode de la longue histoire chahutée de ce couple homme/nature dont le mariage exprime et l'amour et la raison ${ }^{66}$. En effet, le territoire est une entité relationnelle complexe mêlant morphologie de l'espace et subjectivité du regard dans le temps. Si certains auteurs s'affrontent sur la définition du territoire, d'autres suggèrent d'abandonner cette notion polysémique qui ne ferait plus sens. Le territoire constitue en effet un nœud théorique que nous tentons ici de dénouer par la proposition du système socio-patrimonial, alliage des interactions entre rapports sociaux (interactions entre humains contemporains) et patrimoniaux (liens des acteurs, l'espace et au temps). Comment s'articulent rapports social et patrimonial autour des relations et pratiques humaines liées à un bien commun environnemental? Nous proposons un modèle conceptuel d'analyse intitulé l'Acteur en 4 Dimensions, un outil

66 Alberto Magnaghi, Le projet local, Liège, Mardaga, 2003. 
qui vise l'analyse du jeu d'acteurs par l'étude des liens entre humains ainsi que des liens homme-nature. L'A4D permet de constituer les empreintes territoriales, une radiographie synchronique des acteurs dans leurs liens sociaux et patrimoniaux.

L'A4D a été expérimenté sur 4 terrains d'étude différents. Nos résultats montrent que l'étude des liens sociaux ne peut se faire indépendamment du contexte dans lequel ils sont ancrés et, inversement, que les liens homme-nature existent par le biais de relations sociales. Les empreintes territoriales, sources d'informations sur le jeu d'acteurs et de dialogue entre parties intéressées, nous informent également sur les objets du territoire qui font et défont du lien social, sur les interactions entre rapport social et patrimonial, sur les visions de la concertation et de la conservation idéale, sur le degré d'attachement et d'éloignement des acteurs au territoire. Bref, le modèle de l'A4D se propose de décortiquer cette notion complexe qu'est le territoire au travers d'une représentation simplifiée des rapports sociaux et patrimoniaux. Un médiateur territorial ou un porteur de projet peut alors cerner les acteurs forts et faibles, les conflits d'usage ou de valeurs morales, les porte-voix d'acteurs faibles ou les porte-parole d'entités environnementales; il pourra également adapter la négociation territoriale à la présence ou non d'acteurs de type consensuellement correct, protecteur conflictuel, petit cubique ou dominateur distant.

Il y a quand même quelque frustration à n'avoir pu étudier davantage certains sujets qui l'auraient mérité. La notion d'attachement, d'abord, ressort systématiquement des entretiens, sous diverses formes : nostalgie, intérêt, passion... Tous les représentants de groupe rencontrés avouent aimer une tradition, un paysage, un bout de rivière. L'attachement se rapprochant de la notion de sensibilité environnementale, il est intéressant d'analyser de quelle manière il se construit et se manifeste sur le territoire, quels en sont les effets sur le milieu comme sur le complexe. C'est une piste de recherche qui nous apparait potentiellement riche d'enseignements. Par ailleurs, les liens entre nature et culture représentent un thème certes abordé dans notre étude, mais qui mérite un approfondissement notable. Notre modèle 
tente d'approcher les relations existantes entre rapport social et patrimonial, mais ne parvient qu'à les effleurer. L'encastrement des sociétés et des natures représente de plus en plus un objet de la littérature anthropologique et sociologique. Construction sociale de la nature, construction naturelle de la culture, certains parlent d'anthroposystème comme d'un système interactif entre sociosystème et écosystème. À l'échelle d'un territoire, il a été intéressant de tenter d'observer la co-évolution des liens entre humains et des liens aux non-humains. Quels objets font (ou défont) du lien social? Quels liens sociaux font (ou défont) des objets? Des questionnements qui apparaissent fondamentaux dans la recherche sur les attaches entre nature et culture.

\section{Bibliographie}

Barel, Yves, La société du vide, Paris, Seuil, coll. «Empreintes», 1984.

Barouch, Georges, La décision en miettes. Système de pensée et d'action à l'ouvre dans les milieux naturels, Paris, L'Harmattan, coll. "Logiques sociales", 1989.

Benko, Georges, "Territoires et sciences sociales», dans Xabier Itçaina, Jacques Palard et Sébastien Ségas (dir.), Régimes territoriaux et développement économique, Rennes, Presses universitaires de Rennes, 2007, p. 105-112.

Bertrand, Georges, "Paysages et géographie physique globale», RGPSO, tome 39, fasc. 3, 1968, p. 249-272.

Biarez, Sylvie, «Pouvoirs et organisations locales : vers un nouveau paradigme politique", Sciences de la société, no 38, 1996, p. 23-46.

Bourque, Roger et Christian Thuderoz Sociologie de la négociation, Paris, La Découverte et Syros, 2002.

Cattan, Nadine, "Gendering Mobility: Insights into the Construction of Spatial Concepts", dans Tanu Prya Uteng et Tim Cresswell (dir.), Gendered Mobilities, Aldershot, Ashgate, 2008, p. 83-98.

Certeau, Michel de, "L'opération historique», dans Jacques Le Goff et Pierre Nora (dir.), Faire de l'Histoire, vol. 1, Nouveaux problèmes, Gallimard, NRF, 1974. 
Chastel, Alain, "Le Patrimoine», dans Symposium les enjeux, Paris, Encyclopædia Universalis, 1988, p. 267-274.

Crozier, Michel et Ehrard Friedberg, L'acteur et le système, Paris, Seuil, 1977. Dardel, Éric, L’homme et la terre, Paris, vol. 6, Comité des travaux historiques et scientifiques, 1990.

Debarbieux, Bernard, «Territoire», dans Jacques Lévy et Michel Lussault (dir.), Dictionnaire de la géographie et de l'espace des sociétés, Paris, Belin, 2003.

Di Méo, Guy, L’homme, la société, l'espace, Paris, Anthropos, 1990.

Di Méo, Guy, Géographie sociale et territoire, Paris, Nathan, 1998.

Donnadieu, Gérard, La systémique : penser et agir dans la complexité, Rueil-Malmaison, Liaisons, 2002.

Faure, Alain, «Territoires/territorialisation", dans Laurie Boussaget, Sophie Jaquot et Pauline Ravinet (dir.), Dictionnaire des politiques publiques, Paris, Presses de Sciences Po, 2004.

François, Hugues, «Territoire et patrimoine : la co-construction d'une dynamique et de ses ressources", Revue d'économie régionale et urbaine, $\mathrm{n}^{\circ}$ 5, 2006, p. 683-700.

Friedberg, Erhard, Le pouvoir et la règle, Paris, Seuil, coll. «Sociologie», 1993.

Giraut, Frédéric, "Conceptualiser le territoire», Historiens et géographes, ${ }^{\circ}$ 403, 2008, p. 57-68.

Godelier, Maurice, Lidéal et le matériel, Paris, Fayard, 1984.

Godet, Michel, Manuel de prospective stratégique, une indiscipline intellectuelle, Paris, Dunod, 2001.

Guérin, Jean-Paul, «Patrimoine, patrimonialisation, enjeux géographiques», dans Jean-Marc Fournier (dir.), Faire la géographie sociale aujourd'hui, Caen, Presses universitaires de Caen, ${ }^{\circ}$ 14, p. 41-48, 2001.

Jolivet, Marie-José, «Logiques identitaires, logiques territoriales», Autrepart, $\mathrm{n}^{\circ} 14,2000$.

Jollivet, Marcel et Alfredo Pena-Vega, «Relier les connaissances, transversalité, interdisciplinarité», Nature, Sciences et sociétés, vol. 10, ${ }^{\circ} 1$, 2002, p. 78-95.

Kuty, Olgierd, La négociation des valeurs : introduction à la sociologie, Bruxelles, coll. "Ouvertures sociologiques», De Boeck, 1998.

Leroy, Philippe, «La sociologie de l'environnement en Europe : évolution, champ d'action et ambivalences", Natures, Sciences et Sociétés, vol. 9, $\mathrm{n}^{\circ} 1,2001$, p. 29-39. 
Lévy, Jacques et Michel Lussault, Dictionnaire de la géographie et de l'espace des sociétés, Paris, Belin, 2003.

Magnaghi, Alberto, Le projet local, Liège, Mardaga, 2003.

Micoud, André, «Entre Loire et Rhône, ou comment des objets naturels peuvent faire du lien", dans André Micoud et Michel Péroni (dir.), Ce qui nous relie, La Tour-d'Aigues, Éditions de l'Aube, 2000, p. 227-239.

Moine, Alexandre, «Le territoire comme un système complexe : un concept opératoire pour l'aménagement et la géographie», L'espace géographique, vol. 2, tome 35, p. 115-132, 2006.

Moles, Abraham, "Vers une psycho-géographie», dans Antoine Bailly, Robert Ferras et Denise Pumain (dir.), Encyclopédie de géographie, Economica, 1995, p. 159-187.

Montgolfier, Jean de, Le patrimoine du futur. Approche pour une gestion patrimoniale des ressources naturelles, Paris, Economica, 1987.

Morin, Edgar, Commune en France, la métamorphose de Plodémet, Paris, Fayard, 1967.

Ollagnon, Henry, «Stratégies patrimoniales pour un développement durable», dans Christian Barrère et al, Patrimoine, approches croisées, $\mathrm{n}^{\circ} 2$, École doctorale des sciences juridiques, économiques et de gestion, 2005.

Ost, François, La nature hors la loi. L'écologie à l'épreuve du droit, Paris, La Découverte, 1995.

Paasi, Aansi, "Region and Place: Regional Identity in Question», Progress in Human Geography, vol. 27, no 4, 2003, p. 475-485.

Paquot, Thierry, "Qu'est-ce qu'un territoire?", Vie sociale, vol. 2, $\mathrm{n}^{\circ}$ 2, 2011, p. 23-32.

Pecqueur, Bernard, Le développement local, $2^{\mathrm{e}}$ édition, Dijon, Syros, 2000.

Pecqueur, Bernard, «De l'exténuation à la sublimation : la notion de territoire est-elle encore utile?», Géographie, économie, société, vol. 11, n 1, 2009, p. 55-62.

Prieur, Michel, Droits de l'environnement, Dalloz, Paris, 1984.

Puech, Damien, «La prise de conscience du caractère patrimonial de l'eau, origine de nouvelles perspectives de gestion de cet éléments naturel?», dans GIP Hydrosystèmes, Chantal Aspe et Patrick Point (dir.), L'eau en représentations : gestion des milieux aquatiques et représentations sociales, CEMAGREF, 1999.

Pupin, Vincent, Les approches patrimoniales au regard de la question de la prise en charge du monde, Paris, Thèse de doctorat, Agro-Paris Tech, 2008. 
Quivy, Raymond et Luc van Campenhoudt, Manuel de recherche en sciences sociales, Paris, Dunod, 1995.

Retaillé, Denis, "L'espace mobile», dans Benoît Antheaume et Frédéric Giraut (dir.), Le territoire est mort. Vive les territoires!, Paris, Éditions de l'IRD, 2005, p. 175-201.

Rey, Alain, Le Robert : dictionnaire historique de la langue française, tomes 1, 2 et 3, Paris, Dictionnaire Le Robert, 1998.

Sébastien, Léa, Humains et non-humains en pourparlers : l'Acteur en 4 Dimensions. Proposition théorique et méthodologique transdisciplinaire favorisant l'émancipation de nouvelles formes de gouvernances environnementales. Application au domaine de l'eau sur trois territoires: la Plaine du Forez, les pentes du Kilimandjaro et les Barthes de l'Adour, Thèse de doctorat, École des Mines de St-Étienne, 2006.

Sébastien, Léa, "Quand les acteurs faibles et absents s'immiscent dans la négociation environnementale ", Territoire en mouvement, $\mathrm{n}^{\circ} 11,2011$, p. 66-81.

Serres, Michel, Le Contrat Naturel, Paris, Flammarion, 1992. 


\section{Annexe}

\section{Présentation des indicateurs d'une sous-dimension de l'A4D}

Dimension Coopération - Sous-dimension Estime Composantes

\begin{tabular}{|c|c|}
\hline $\begin{array}{l}\text { I.1.1. Regard sur les } \\
\text { autres }\end{array}$ & $\begin{array}{l}0: \text { ne parle jamais des autres ni de leurs projets, ou en parle négativement (dimension conflit) } \\
1: \text { parle des autres mais pas de leurs projets, de manière neutre } \\
2: \text { parle des autres et de leurs projets de manière neutre - apprécie uniquement ses partenaires } \\
3: \text { apprécie certains projets mais critique les acteurs qui les portent } \\
4: \text { montre de l'intérêt pour certains acteurs et projets, en plus de ceux de ses partenaires } \\
5: \text { est consensuel, voire enthousiaste face à de nombreux acteurs et projets }\end{array}$ \\
\hline $\begin{array}{l}\text { I.1.2. Respect des } \\
\text { institutions }\end{array}$ & $\begin{array}{l}0: \text { ne parle jamais des textes de loi ni des grands programmes, ou en parle négativement (dimension } \\
\text { conflit) } \\
1: \text { parle des lois ou des grands programmes de manière neutre } \\
2: \text { parle des lois et des grands programmes de manière neutre } \\
3: \text { apprécie certaines lois ou grands programmes } \\
4: \text { apprécie certaines lois et grands programmes } \\
5: \text { participe aux grands programmes ou a une démarche volontaire allant au-devant de la loi }\end{array}$ \\
\hline $\begin{array}{l}\text { I.1.3. Regard sur } \\
\text { l'action commune }\end{array}$ & $\begin{array}{l}0: \text { ne souhaite pas de nouveaux partenaires et critique négativement l'idée de concertation } \\
1: \text { souhaite seulement plus de contacts avec sa sphère proche (clients, administrés, financeurs) pour } \\
\text { répondre uniquement à ses intérêts } \\
2: \text { souhaite la concertation si elle est encadrée par un homme fort, un décideur (lui-même) - demande du } \\
\text { pouvoir } \\
3: \text { souhaite la concertation si elle est encadrée par un homme fort, un décideur (un autre acteur) - } \\
\text { demande de la reconnaissance } \\
4: \text { souhaite la concertation si elle est restreinte aux partis concernés } \\
5: \text { souhaite la concertation ouverte à tous (gouvernance, démarches participatives, palabres africaines) }\end{array}$ \\
\hline $\begin{array}{l}\text { I.1.4. Degré } \\
\text { d'implication }\end{array}$ & $\begin{array}{l}0: \text { n'envisage aucune démarche vers les autres } \\
1: \text { rencontre peu les autres, fait acte de présence, se dit acteur distant - conformité passive } \\
2: \text { rencontre les autres (réunion, conseil technique) pour leur expliquer qu'ils font mal, veut changer les } \\
\text { mentalités } \\
3: \text { rencontre les autres (réunion, conseil technique) pour mieux se faire connaître, expliquer ce qu'il fait } \\
\text { bien, se dit acteur non-entendu } \\
4: \text { participe à des concertations territoriales pour partager, écouter, pour construire ensemble } \\
5: \text { organise des concertations territoriales pour partager, écouter, pour une vision commune - } \\
\text { coopération zélée, se dit acteur liant }\end{array}$ \\
\hline
\end{tabular}

